\title{
Supplementary Information and Figures
}

Crystal structure of magnesium selenate enneahydrate, $\mathrm{MgSeO}_{4} \cdot 9 \mathrm{H}_{2} \mathrm{O}$, from $5-250 \mathrm{~K}$ using neutron time-of-flight Laue diffraction

A. Dominic Fortes ${ }^{1,2, *}$ Dario Alfè, ${ }^{2}$ Eduardo R. Hernández, ${ }^{3}$ and Matthias J. Gutmann ${ }^{4}$

${ }^{1}$ Department of Earth Sciences, University College London, Gower Street, London WC1E 6BT, U.K.

${ }^{2}$ Department of Earth and Planetary Sciences, Birkbeck, University of London, Malet Street, London WC1E 7HX,

U.K.

${ }^{3}$ Instituto de Ciencia de Materiales de Madrid (ICMM-CSIC), Campus de Cantoblanco, 28049 Madrid, Spain

${ }^{4}$ ISIS Facility, Rutherford Appleton Laboratory, Harwell Science and Innovation Campus, Didcot, Oxfordshire OX11 0QX, UK

*Corresponding author email: andrew.fortes@ucl.ac.uk

\section{CONTENTS}

1. Characterization of $\mathrm{MgSeO}_{4} \cdot 6 \mathrm{H}_{2} \mathrm{O}$ (p. 2)

2. Ab initio atomic coordinates $\mathrm{MgSeO}_{4} \cdot 9 \mathrm{H}_{2} \mathrm{O}$ (p. 5)

3. Raman spectroscopic data (p. 7) 


\section{Supplementary Information and Figures}

\section{Characterization of $\mathrm{MgSeO}_{4} \cdot 6 \mathrm{H}_{2} \mathrm{O}$}

Since we report a hitherto unknown hydration state of a compound that had to be synthesised from scratch, we wish to ensure absolute transparency with regard to our characterization of the starting materials. In particular, it is important to show that the crystalline material produced by reaction of $\mathrm{MgO}$ with aqueous $\mathrm{H}_{2} \mathrm{SeO}_{4}$ and subsequent evaporation was indeed $\mathrm{MgSeO}_{4} \cdot 6 \mathrm{H}_{2} \mathrm{O}$, a compound that has previously been characterized by $\mathrm{X}$-ray single-crystal diffraction methods.

A single crystal of approximate dimensions $5 \times 5 \times 2 \mathrm{~mm}$ (Figure S1) was powdered and back-loaded into a standard spinner sample holder. This powder specimen was measured in the range $5-150^{\circ} 2 \theta$ over a period of $21 \mathrm{hr}$ using a PANalytical X'Pert Pro powder diffractometer (Ge monochromated radiation, $\lambda=\mathrm{Co} K \alpha_{1}=1.788996 \AA$ ). The data were fitted by the Rietveld method in GSAS/Expgui to yield the unit-cell parameters listed in the first column of Table S1.

The unit-cell parameters are in very good agreement with Kolitsch (2002), the volume being $5.1 \%$ larger than the isotypic sulfate crystal (column 3, Table S1). There are no discernible peaks from any accessory phase in the powder diffraction data (Figures S2 and S3); in other words, the material used to prepare the enneahydrate described in the main body of this work was prepared from phase-pure $\mathrm{MgSeO}_{4} \cdot 6 \mathrm{H}_{2} \mathrm{O}$.

\section{Supplementary Figure S1}

Photograph of a representative single crystal of $\mathrm{MgSeO}_{4} \cdot 6 \mathrm{H}_{2} \mathrm{O}$ (left) and an indexed drawing - prepared using WinXMorph - that shows the faces present (right). The crystal morphology agrees well with that described by Von Groth (1908).
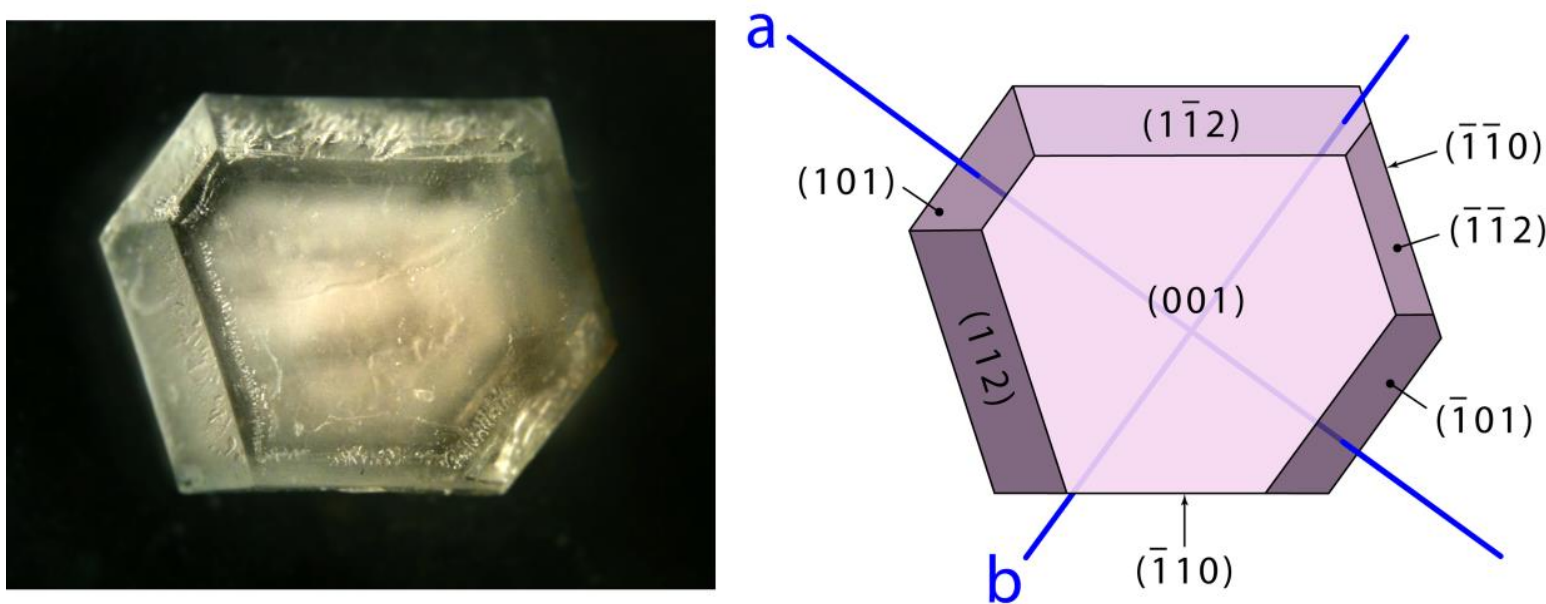


\section{Supplementary Information and Figures}

\section{Supplementary Table S1}

Unit-cell parameters of $\mathrm{MgSeO}_{4} \cdot 6 \mathrm{H}_{2} \mathrm{O}$ compared with the isotypic crystal $\mathrm{MgSO}_{4} \cdot 6 \mathrm{H}_{2} \mathrm{O}$.

\begin{tabular}{|l|cc|cc|}
\cline { 2 - 5 } \multicolumn{1}{c|}{} & \multicolumn{2}{c|}{$\mathrm{MgSeO}_{4} \cdot 6 \mathrm{H}_{2} \mathrm{O}$} & \multicolumn{2}{c|}{$\mathrm{MgSO}_{4} \cdot 6 \mathrm{H}_{2} \mathrm{O}$} \\
& This work & Kolitsch (2002) & Zalkin et al. (1964) & Batsanov (2000) \\
& $298 \mathrm{~K}$ & $293 \mathrm{~K}$ & "room T" & $120 \mathrm{~K}$ \\
\hline$a(\AA)$ & $10.21875(9)$ & $10.224(1)$ & $10.110(5)$ & $9.975(2)$ \\
$b(\AA)$ & $7.36537(7)$ & $7.370(1)$ & $7.212(4)$ & $7.186(2)$ \\
$c(\AA)$ & $24.8538(3)$ & $24.866(2)$ & $24.41(1)$ & $24.267(6)$ \\
$\beta\left({ }^{\circ}\right)$ & $98.4087(3)$ & $98.41(1)$ & $98.30(5)$ & $98.78(1)$ \\
$\mathrm{V}(\AA)$ & $1850.50(5)$ & $1853.5(3)$ & $1761(1)$ & $1719.1(7)$ \\
\hline
\end{tabular}

\section{Supplementary Figure S2}

X-ray diffraction pattern of powdered single crystals of $\mathrm{MgSeO}_{4} \cdot 6 \mathrm{H}_{2} \mathrm{O}$, measured at room temperature. The red points report the measured data, the green line represents a Rietveld refinement and the pink line underneath is a difference profile. Note the use of a square root intensity scale in order to emphasise weaker peaks.

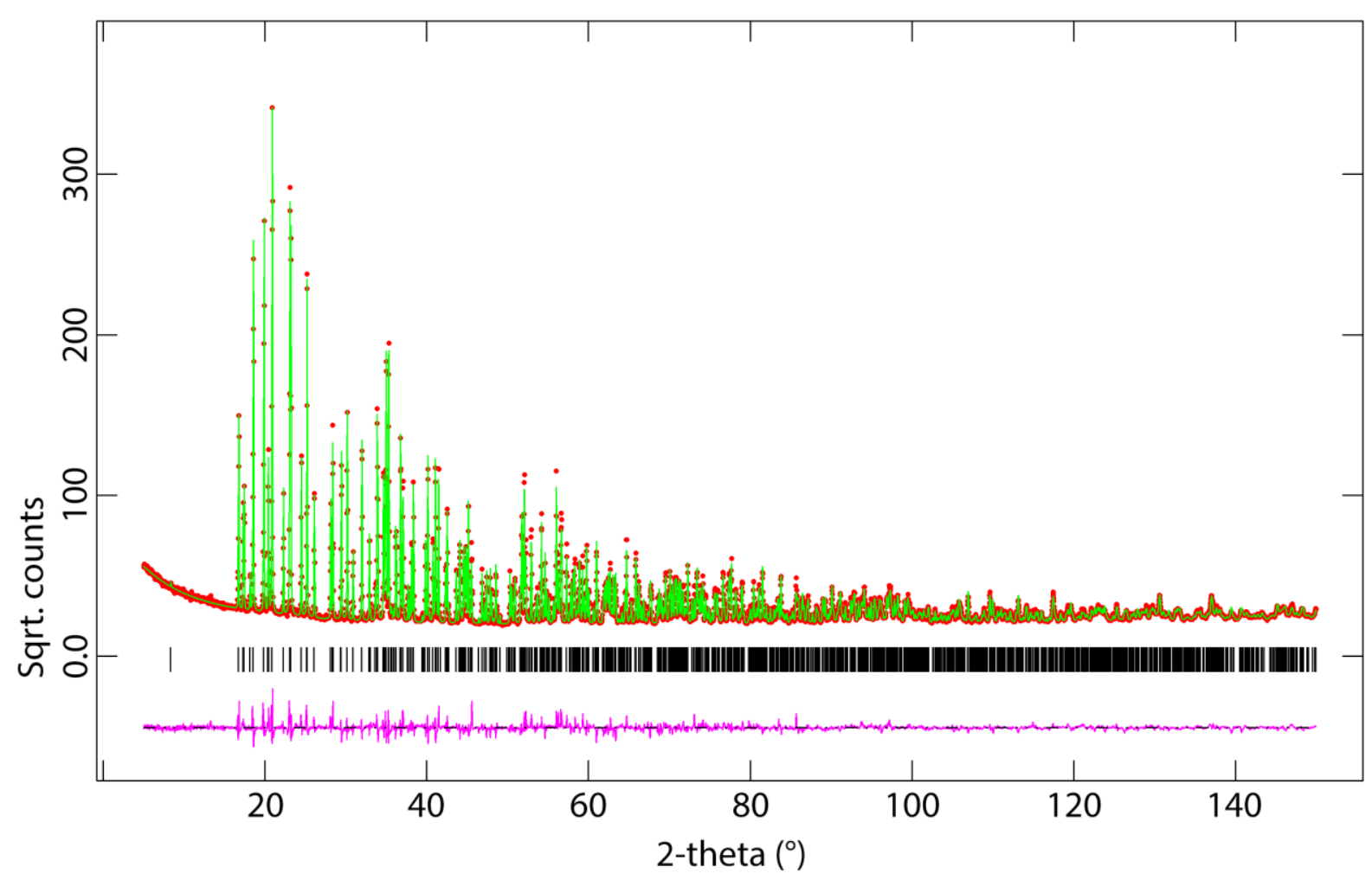




\section{Supplementary Information and Figures}

\section{Supplementary Figure S3}

Expanded portion of Figure S1, showing the low $2 \theta$ range where peaks are well dispersed. This shows very clearly that there are no accessory phases present; the use of a square root intensity scale is designed to emphasise even the weakest of possible parasitic peaks.

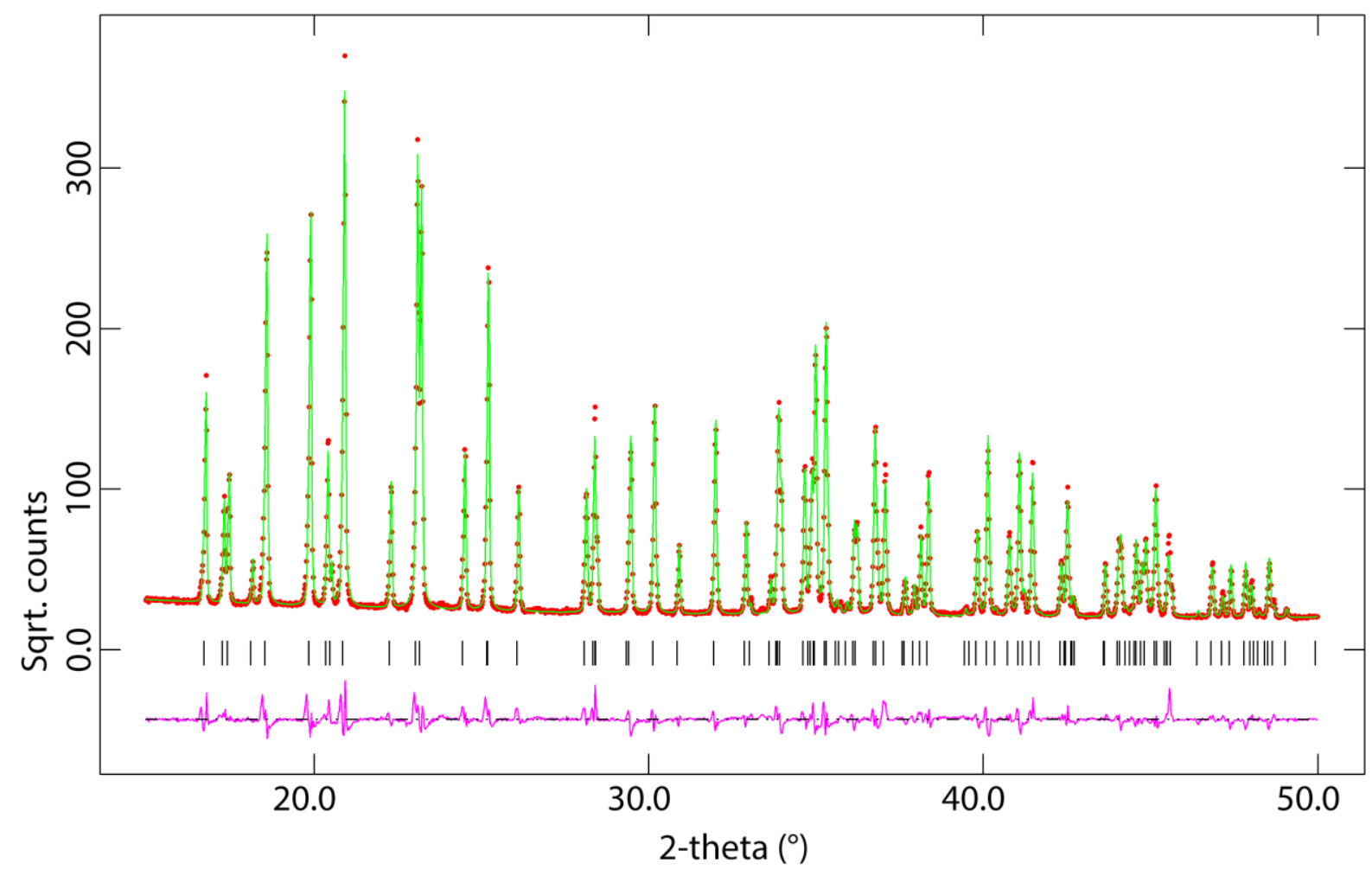

\section{References}

Batsanov, A. S. (2000) Acta Crystallogr. C 56, e230-e231.

Kolitsch, U. (2002) Acta Crystallogr. E 58, i3-i5.

Von Groth, P. (1908) Chemische Krystallographie, vol. 2, p423.

Zalkin, A., Ruben, H. \& Templeton, D. H. (1964). Acta Crystallgr. 17, 235-240. 


\section{Supplementary Information and Figures}

\section{2. $A b$ initio atomic coordinates $\mathrm{MgSeO}_{4} \cdot 9 \mathrm{H}_{2} \mathrm{O}$}

\section{Supplementary Table S2}

Unit-cell parameters and fractional atomic coordinates of $\mathrm{MgSeO}_{4} \cdot 9 \mathrm{H}_{2} \mathrm{O}$ as determined by $a b$ initio DFT calculations at zero pressure and temperature.

\begin{tabular}{|c|c|c|c|}
\hline \multicolumn{4}{|c|}{$\begin{array}{l}\text { Space-group } \mathrm{P} 2{ }_{1} / c \\
a=7.280131 \AA \\
b=10.505192 \AA \\
c=17.256095 \AA \\
\beta=109.254973^{\circ}\end{array}$} \\
\hline Atom & $x$ & $y$ & $z$ \\
\hline $\mathrm{Se}$ & 0.60747 & 0.44527 & 0.33233 \\
\hline $\mathrm{O} 1$ & 0.67257 & 0.29611 & 0.32243 \\
\hline $\mathrm{O} 2$ & 0.37347 & 0.44991 & 0.32742 \\
\hline $\mathrm{O} 3$ & 0.63624 & 0.53669 & 0.25750 \\
\hline $\mathrm{O} 4$ & 0.74514 & 0.50394 & 0.42365 \\
\hline $\mathrm{Mg}$ & 0.89995 & 0.29687 & 0.12261 \\
\hline Ow1 & 0.15705 & 0.26082 & 0.21893 \\
\hline Ow2 & 0.04907 & 0.38100 & 0.05462 \\
\hline Ow3 & 0.91367 & 0.11704 & 0.07198 \\
\hline Ow4 & 0.72000 & 0.21251 & 0.18029 \\
\hline Ow5 & 0.89739 & 0.47269 & 0.17704 \\
\hline Ow6 & 0.63033 & 0.33646 & 0.02608 \\
\hline Ow7 & 0.97380 & 0.68180 & 0.09987 \\
\hline Ow8 & 0.65789 & 0.83569 & 0.03513 \\
\hline Ow9 & 0.40978 & 0.49141 & 0.09444 \\
\hline H9B & 0.49724 & 0.43224 & 0.07832 \\
\hline H9A & 0.47837 & 0.51296 & 0.15259 \\
\hline H8B & 0.67563 & 0.89352 & 0.99295 \\
\hline $\mathrm{H} 8 \mathrm{~A}$ & 0.64744 & 0.88902 & 0.08110 \\
\hline H7B & 0.86555 & 0.74440 & 0.08148 \\
\hline H7A & 0.09374 & 0.73065 & 0.12750 \\
\hline H6B & 0.62364 & 0.39170 & 0.97804 \\
\hline H6A & 0.53146 & 0.26807 & 0.00449 \\
\hline $\mathrm{H} 5 \mathrm{~B}$ & 0.92635 & 0.55222 & 0.15181 \\
\hline $\mathrm{H} 5 \mathrm{~A}$ & 0.82004 & 0.49640 & 0.21194 \\
\hline H4B & 0.70536 & 0.11928 & 0.17998 \\
\hline $\mathrm{H} 4 \mathrm{~A}$ & 0.69705 & 0.24382 & 0.23036 \\
\hline H3B & 0.04319 & 0.07965 & 0.07905 \\
\hline $\mathrm{H} 3 \mathrm{~A}$ & 0.83635 & 0.09329 & 0.01537 \\
\hline
\end{tabular}


Supplementary Information and Figures

\begin{tabular}{|l|l|l|l|}
\hline H2B & 0.17952 & 0.42044 & 0.07559 \\
\hline H2A & 0.02707 & 0.36376 & 0.99561 \\
\hline H1B & 0.22925 & 0.32942 & 0.25515 \\
\hline H1A & 0.24611 & 0.18808 & 0.22522 \\
\hline
\end{tabular}




\section{Supplementary Information and Figures}

\section{Raman spectroscopic data}

\section{Supplementary Table S3}

Raman spectroscopic data acquired from single crystals of $\mathrm{MgSeO}_{4}$ hydrates at various temperatures, and from powdered $\beta-\mathrm{MgSeO}_{4}$. Intensities are in arbitrary units.

\begin{tabular}{|c|c|c|c|c|}
\hline $\begin{array}{l}\text { Raman shift } \\
\left(\mathrm{cm}^{-1}\right)\end{array}$ & $\begin{array}{c}\mathrm{MgSeO}_{4} \cdot 9 \mathrm{H}_{2} \mathrm{O} \\
259 \mathrm{~K}\end{array}$ & $\begin{array}{c}\mathrm{MgSeO}_{4} \cdot 9 \mathrm{H}_{2} \mathrm{O} \\
78 \mathrm{~K}\end{array}$ & $\begin{array}{c}\mathrm{MgSeO}_{4} \cdot 6 \mathrm{H}_{2} \mathrm{O} \\
\text { Room-T }\end{array}$ & $\begin{array}{c}\beta-\mathrm{MgSeO}_{4} \\
\text { Room- }\end{array}$ \\
\hline 171.23 & 583.97 & 345.78 & 50.00 & 1914.10 \\
\hline 174.09 & 655.98 & 457.21 & 16.61 & 1520.77 \\
\hline 176.95 & 661.60 & 357.08 & 23.50 & 903.73 \\
\hline 179.81 & 678.98 & 317.88 & 74.58 & 493.05 \\
\hline 182.67 & 725.39 & 314.56 & 81.46 & 318.34 \\
\hline 185.53 & 675.86 & 155.08 & 130.40 & 221.53 \\
\hline 188.39 & 646.44 & 268.90 & 241.18 & 133.95 \\
\hline 191.24 & 629.14 & 229.32 & 303.41 & 113.73 \\
\hline 194.09 & 677.16 & 347.76 & 422.80 & 72.69 \\
\hline 196.95 & 764.19 & 410.56 & 510.53 & 63.38 \\
\hline 199.80 & 836.03 & 391.41 & 609.69 & 100.00 \\
\hline 202.65 & 919.79 & 193.73 & 674.74 & 210.75 \\
\hline 205.50 & 1046.36 & 463.20 & 831.49 & 343.39 \\
\hline 208.35 & 1128.74 & 537.64 & 943.00 & 488.57 \\
\hline 211.20 & 1224.28 & 600.75 & 1059.77 & 738.59 \\
\hline 214.05 & 1255.29 & 580.90 & 1127.31 & 971.95 \\
\hline 216.89 & 1252.30 & 769.45 & 1201.09 & 1241.55 \\
\hline 219.74 & 1190.43 & 958.86 & 1247.05 & 1541.41 \\
\hline 222.58 & 1191.47 & 1066.59 & 1219.99 & 1803.05 \\
\hline 225.42 & 1151.46 & 1209.74 & 1255.89 & 1831.54 \\
\hline 228.27 & 1167.82 & 1086.01 & 1247.18 & 1674.55 \\
\hline 231.11 & 1215.24 & 1070.53 & 1256.23 & 1296.05 \\
\hline 233.95 & 1196.11 & 1179.31 & 1272.45 & 871.44 \\
\hline 236.79 & 1207.59 & 1156.12 & 1259.17 & 573.29 \\
\hline 239.62 & 1160.47 & 1189.08 & 1269.16 & 355.94 \\
\hline 242.46 & 1031.47 & 1141.93 & 1266.16 & 237.55 \\
\hline 245.30 & 887.27 & 1203.18 & 1261.09 & 165.97 \\
\hline 248.13 & 778.22 & 1249.38 & 1227.90 & 129.37 \\
\hline 250.97 & 666.59 & 1162.24 & 1183.08 & 136.31 \\
\hline 253.80 & 550.70 & 852.38 & 1060.20 & 103.58 \\
\hline 256.63 & 454.62 & 532.97 & 904.42 & 129.94 \\
\hline 259.46 & 388.75 & 440.79 & 753.73 & 169.62 \\
\hline 262.29 & 299.43 & 452.22 & 623.66 & 206.34 \\
\hline 265.12 & 265.16 & 542.68 & 528.22 & 266.07 \\
\hline
\end{tabular}




\section{Supplementary Information and Figures}

\begin{tabular}{|c|c|c|c|c|}
\hline 267.95 & 232.39 & 392.33 & 424.74 & 291.00 \\
\hline 270.77 & 189.33 & 475.59 & 409.03 & 381.62 \\
\hline 273.60 & 185.07 & 263.47 & 358.43 & 470.19 \\
\hline 276.43 & 137.81 & 233.10 & 362.11 & 557.78 \\
\hline 279.25 & 119.20 & 283.52 & 376.91 & 606.28 \\
\hline 282.07 & 53.39 & 353.30 & 406.53 & 648.08 \\
\hline 284.89 & 79.63 & 353.14 & 393.68 & 554.86 \\
\hline 287.71 & 101.82 & 299.58 & 389.61 & 403.39 \\
\hline 290.53 & 170.41 & 198.60 & 398.46 & 277.22 \\
\hline 293.35 & 219.25 & 231.43 & 448.54 & 169.54 \\
\hline 296.17 & 287.93 & 296.21 & 464.54 & 119.06 \\
\hline 298.99 & 404.72 & 161.25 & 473.63 & 109.53 \\
\hline 301.80 & 431.95 & 406.86 & 550.36 & 148.24 \\
\hline 304.62 & 524.28 & 448.24 & 607.82 & 212.44 \\
\hline 307.43 & 563.20 & 507.08 & 666.99 & 266.02 \\
\hline 310.25 & 641.27 & 666.16 & 760.70 & 370.70 \\
\hline 313.06 & 777.19 & 833.65 & 847.35 & 550.33 \\
\hline 315.87 & 860.35 & 951.65 & 973.18 & 770.36 \\
\hline 318.68 & 1034.45 & 931.48 & 1088.36 & 1108.85 \\
\hline 321.49 & 1253.15 & 826.94 & 1243.14 & 1566.46 \\
\hline 324.29 & 1513.39 & 836.33 & 1438.71 & 2169.60 \\
\hline 327.10 & 1880.58 & 957.76 & 1668.09 & 2865.81 \\
\hline 329.91 & 2286.96 & 1106.02 & 1928.66 & 3739.49 \\
\hline 332.71 & 2713.88 & 1403.77 & 2231.29 & 4615.63 \\
\hline 335.52 & 3199.55 & 2033.40 & 2599.19 & 5446.50 \\
\hline 338.32 & 3724.05 & 2599.92 & 2950.66 & 6083.83 \\
\hline 341.12 & 4369.90 & 3125.82 & 3273.35 & 6284.87 \\
\hline 343.92 & 4921.34 & 3619.83 & 3622.09 & 5808.76 \\
\hline 346.72 & 5449.02 & 3997.43 & 3851.85 & 4739.87 \\
\hline 349.52 & 5801.58 & 4759.12 & 4077.96 & 3827.80 \\
\hline 352.32 & 6131.84 & 5697.56 & 4126.72 & 3435.01 \\
\hline 355.12 & 6269.74 & 6211.66 & 4111.37 & 3667.55 \\
\hline 357.91 & 6326.92 & 6663.29 & 4097.68 & 4362.29 \\
\hline 360.71 & 6163.60 & 6824.15 & 4099.35 & 5424.86 \\
\hline 363.50 & 5638.16 & 6654.50 & 4089.03 & 6695.79 \\
\hline 366.29 & 4897.21 & 6657.99 & 4101.26 & 8065.73 \\
\hline 369.08 & 4051.84 & 6732.58 & 4073.64 & 9418.48 \\
\hline 371.88 & 3267.96 & 6551.44 & 4007.04 & 10169.88 \\
\hline 374.67 & 2718.22 & 5328.91 & 3772.79 & 9427.61 \\
\hline 377.45 & 2362.56 & 3747.34 & 3482.36 & 7302.18 \\
\hline 380.24 & 2164.54 & 2673.21 & 3175.03 & 5047.17 \\
\hline 383.03 & 2121.76 & 2239.33 & 2886.65 & 3877.50 \\
\hline 385.82 & 2253.96 & 2080.51 & 2632.62 & 3585.96 \\
\hline 388.60 & 2552.69 & 2165.33 & 2499.95 & 3920.74 \\
\hline
\end{tabular}




\section{Supplementary Information and Figures}

\begin{tabular}{|c|c|c|c|c|}
\hline 391.38 & 3027.00 & 2400.17 & 2419.63 & 4812.13 \\
\hline 394.17 & 3651.65 & 3088.76 & 2488.71 & 6297.30 \\
\hline 396.95 & 4256.08 & 3789.03 & 2608.88 & 8453.31 \\
\hline 399.73 & 4962.55 & 4348.75 & 2743.14 & 11415.13 \\
\hline 402.51 & 5514.74 & 5163.25 & 2967.60 & 15147.63 \\
\hline 405.29 & 5906.37 & 5902.93 & 3207.85 & 19303.81 \\
\hline 408.07 & 5823.08 & 6819.13 & 3457.68 & 23177.67 \\
\hline 410.84 & 5393.57 & 7462.74 & 3674.13 & 25243.94 \\
\hline 413.62 & 4995.79 & 7268.85 & 3878.35 & 25655.29 \\
\hline 416.40 & 4751.74 & 5814.86 & 4044.98 & 25101.84 \\
\hline 419.17 & 4809.72 & 4721.54 & 4181.07 & 21270.86 \\
\hline 421.94 & 4810.37 & 4643.56 & 4327.14 & 15348.44 \\
\hline 424.72 & 4690.81 & 5134.80 & 4419.30 & 11327.97 \\
\hline 427.49 & 4501.03 & 5628.43 & 4479.40 & 9112.99 \\
\hline 430.26 & 4005.02 & 5795.08 & 4484.50 & 7373.34 \\
\hline 433.03 & 3421.74 & 5874.70 & 4481.65 & 5452.92 \\
\hline 435.79 & 2761.74 & 5564.95 & 4330.33 & 3635.54 \\
\hline 438.56 & 2211.56 & 5110.70 & 4196.13 & 2422.15 \\
\hline 441.33 & 1803.76 & 3941.90 & 3940.74 & 1648.71 \\
\hline 444.09 & 1489.04 & 2778.86 & 3690.81 & 1207.93 \\
\hline 446.86 & 1208.39 & 2220.32 & 3382.11 & 912.20 \\
\hline 449.62 & 1014.97 & 1792.29 & 3052.34 & 694.24 \\
\hline 452.38 & 867.57 & 1723.95 & 2757.47 & 530.80 \\
\hline 455.14 & 710.10 & 1520.73 & 2443.38 & 419.54 \\
\hline 457.90 & 606.55 & 1307.64 & 2141.10 & 321.60 \\
\hline 460.66 & 519.07 & 1130.92 & 1847.53 & 280.90 \\
\hline 463.42 & 448.17 & 1032.68 & 1588.56 & 185.99 \\
\hline 466.18 & 414.74 & 1074.65 & 1395.41 & 178.52 \\
\hline 468.94 & 370.63 & 1088.98 & 1209.59 & 141.26 \\
\hline 471.69 & 302.90 & 1075.05 & 1039.17 & 125.70 \\
\hline 474.44 & 314.83 & 1098.18 & 923.12 & 122.20 \\
\hline 477.20 & 222.19 & 1042.84 & 785.22 & 142.41 \\
\hline 479.95 & 255.22 & 791.44 & 654.29 & 202.85 \\
\hline 482.70 & 213.76 & 863.21 & 595.46 & 256.96 \\
\hline 485.45 & 203.08 & 792.91 & 521.07 & 367.85 \\
\hline 488.20 & 196.72 & 756.12 & 453.29 & 479.06 \\
\hline 490.95 & 181.23 & 638.42 & 405.98 & 616.76 \\
\hline 493.70 & 190.21 & 758.27 & 386.71 & 697.47 \\
\hline 496.44 & 185.70 & 717.87 & 334.70 & 630.38 \\
\hline 499.19 & 170.56 & 616.88 & 290.74 & 507.55 \\
\hline 501.94 & 130.69 & 540.82 & 259.12 & 349.67 \\
\hline 504.68 & 137.79 & 482.22 & 233.45 & 265.06 \\
\hline 507.42 & 128.86 & 490.09 & 219.46 & 236.50 \\
\hline 510.16 & 129.49 & 324.49 & 184.61 & 275.35 \\
\hline
\end{tabular}




\section{Supplementary Information and Figures}

\begin{tabular}{|c|c|c|c|c|}
\hline 512.90 & 146.94 & 399.12 & 176.19 & 339.51 \\
\hline 515.64 & 115.95 & 363.71 & 170.85 & 420.35 \\
\hline 518.38 & 88.73 & 309.42 & 149.67 & 588.49 \\
\hline 521.12 & 102.43 & 272.36 & 141.57 & 803.40 \\
\hline 523.86 & 96.24 & 363.94 & 149.43 & 1060.53 \\
\hline 526.59 & 123.36 & 362.42 & 114.82 & 1333.18 \\
\hline 529.33 & 105.25 & 423.91 & 119.46 & 1478.59 \\
\hline 532.06 & 86.80 & 409.86 & 90.25 & 1526.54 \\
\hline 534.79 & 89.17 & 581.16 & 113.68 & 1391.95 \\
\hline 537.53 & 101.25 & 654.36 & 94.53 & 1065.60 \\
\hline 540.26 & 99.64 & 706.57 & 93.33 & 748.97 \\
\hline 542.99 & 98.49 & 727.90 & 72.10 & 514.83 \\
\hline 545.72 & 94.01 & 680.49 & 59.47 & 358.01 \\
\hline 548.44 & 75.53 & 773.55 & 65.80 & 276.65 \\
\hline 551.17 & 74.17 & 575.29 & 37.58 & 244.05 \\
\hline 553.90 & 115.32 & 657.23 & 65.25 & 180.65 \\
\hline 556.62 & 52.68 & 517.06 & 50.28 & 202.34 \\
\hline 559.35 & 81.30 & 378.22 & 25.77 & 155.57 \\
\hline 562.07 & 67.73 & 388.95 & 44.95 & 139.09 \\
\hline 564.79 & 67.02 & 520.59 & 50.80 & 131.33 \\
\hline 567.51 & 58.19 & 443.62 & 36.17 & 104.15 \\
\hline 570.23 & 56.92 & 485.44 & 43.09 & 108.69 \\
\hline 572.95 & 35.74 & 605.38 & 40.12 & 123.22 \\
\hline 575.67 & 41.38 & 685.21 & 71.34 & 103.19 \\
\hline 578.39 & 69.50 & 704.76 & 43.75 & 153.87 \\
\hline 581.11 & 64.07 & 700.90 & 41.01 & 107.67 \\
\hline 583.82 & 40.37 & 693.85 & 79.99 & 120.15 \\
\hline 586.54 & 33.82 & 544.52 & 51.41 & 108.83 \\
\hline 589.25 & 54.22 & 521.44 & 60.52 & 117.46 \\
\hline 591.96 & 87.73 & 409.43 & 63.17 & 102.26 \\
\hline 594.67 & 69.99 & 312.08 & 47.54 & 136.26 \\
\hline 597.38 & 44.26 & 280.72 & 58.42 & 119.15 \\
\hline 600.09 & 102.33 & 363.46 & 91.80 & 127.27 \\
\hline 602.80 & 57.02 & 167.03 & 56.31 & 87.14 \\
\hline 605.51 & 83.86 & 297.59 & 85.13 & 140.47 \\
\hline 608.22 & 99.37 & 370.21 & 93.71 & 144.33 \\
\hline 610.92 & 79.68 & 385.95 & 118.51 & 132.61 \\
\hline 613.63 & 85.40 & 350.34 & 114.06 & 129.41 \\
\hline 616.33 & 108.43 & 378.93 & 117.53 & 108.00 \\
\hline 619.03 & 136.77 & 333.95 & 109.24 & 113.43 \\
\hline 621.74 & 98.01 & 283.21 & 100.08 & 168.98 \\
\hline 624.44 & 90.91 & 388.96 & 137.35 & 142.40 \\
\hline 627.14 & 120.77 & 381.57 & 118.52 & 131.96 \\
\hline 629.84 & 103.38 & 490.65 & 137.82 & 129.74 \\
\hline
\end{tabular}




\section{Supplementary Information and Figures}

\begin{tabular}{|c|c|c|c|c|}
\hline 632.53 & 127.46 & 366.50 & 113.68 & 122.90 \\
\hline 635.23 & 134.68 & 469.14 & 135.42 & 148.55 \\
\hline 637.93 & 171.91 & 381.57 & 121.18 & 133.32 \\
\hline 640.62 & 195.51 & 531.06 & 136.60 & 147.70 \\
\hline 643.32 & 180.90 & 524.58 & 158.23 & 158.32 \\
\hline 646.01 & 163.95 & 556.83 & 154.92 & 152.00 \\
\hline 648.70 & 184.65 & 545.87 & 136.27 & 142.45 \\
\hline 651.39 & 182.81 & 598.09 & 147.68 & 150.36 \\
\hline 654.08 & 204.43 & 495.38 & 133.20 & 133.79 \\
\hline 656.77 & 253.19 & 474.62 & 112.17 & 132.58 \\
\hline 659.46 & 263.81 & 494.96 & 123.02 & 128.98 \\
\hline 662.15 & 237.24 & 609.69 & 136.55 & 147.74 \\
\hline 664.84 & 303.47 & 625.52 & 145.36 & 144.46 \\
\hline 667.52 & 293.30 & 639.92 & 138.35 & 139.77 \\
\hline 670.21 & 322.43 & 746.45 & 172.35 & 123.10 \\
\hline 672.89 & 290.92 & 778.59 & 170.83 & 136.41 \\
\hline 675.57 & 350.06 & 577.87 & 134.55 & 108.18 \\
\hline 678.25 & 400.25 & 669.06 & 144.29 & 135.99 \\
\hline 680.94 & 347.59 & 706.09 & 155.04 & 103.41 \\
\hline 683.62 & 412.29 & 600.77 & 132.21 & 143.51 \\
\hline 686.29 & 390.84 & 693.75 & 155.27 & 133.69 \\
\hline 688.97 & 424.49 & 850.45 & 171.47 & 136.68 \\
\hline 691.65 & 424.14 & 742.53 & 150.49 & 112.15 \\
\hline 694.33 & 458.34 & 974.25 & 177.95 & 159.91 \\
\hline 697.00 & 461.24 & 1033.17 & 174.16 & 155.84 \\
\hline 699.67 & 458.49 & 1096.88 & 184.00 & 154.11 \\
\hline 702.35 & 456.74 & 1223.64 & 217.04 & 171.37 \\
\hline 705.02 & 474.94 & 1099.65 & 195.40 & 173.45 \\
\hline 707.69 & 490.99 & 1175.56 & 222.16 & 213.56 \\
\hline 710.36 & 508.44 & 1050.95 & 223.51 & 159.25 \\
\hline 713.03 & 472.14 & 985.28 & 268.29 & 204.66 \\
\hline 715.70 & 527.24 & 951.28 & 254.56 & 220.60 \\
\hline 718.37 & 534.29 & 758.62 & 253.86 & 264.46 \\
\hline 721.03 & 523.18 & 806.75 & 281.45 & 284.92 \\
\hline 723.70 & 565.53 & 925.23 & 316.34 & 312.53 \\
\hline 726.36 & 483.42 & 914.65 & 343.85 & 308.13 \\
\hline 729.03 & 564.91 & 873.27 & 360.83 & 360.93 \\
\hline 731.69 & 563.24 & 728.79 & 365.33 & 388.91 \\
\hline 734.35 & 564.53 & 871.09 & 412.89 & 431.81 \\
\hline 737.01 & 614.96 & 875.74 & 448.74 & 436.43 \\
\hline 739.67 & 553.69 & 880.95 & 478.32 & 446.10 \\
\hline 742.33 & 616.46 & 723.70 & 480.42 & 466.84 \\
\hline 744.99 & 610.88 & 966.88 & 546.48 & 519.58 \\
\hline 747.65 & 604.65 & 1069.77 & 599.74 & 543.39 \\
\hline
\end{tabular}




\section{Supplementary Information and Figures}

\begin{tabular}{|c|c|c|c|c|}
\hline 750.30 & 585.96 & 1097.03 & 608.02 & 575.27 \\
\hline 752.96 & 588.37 & 1239.86 & 674.53 & 612.38 \\
\hline 755.61 & 613.17 & 1182.43 & 678.42 & 622.12 \\
\hline 758.27 & 603.82 & 1481.56 & 760.38 & 684.94 \\
\hline 760.92 & 589.72 & 1424.98 & 786.96 & 685.93 \\
\hline 763.57 & 604.55 & 1555.47 & 854.63 & 719.06 \\
\hline 766.22 & 599.74 & 1589.88 & 894.33 & 719.20 \\
\hline 768.87 & 590.57 & 1475.79 & 918.15 & 737.78 \\
\hline 771.52 & 628.29 & 1443.50 & 952.70 & 774.10 \\
\hline 774.17 & 629.60 & 1275.03 & 966.57 & 829.87 \\
\hline 776.81 & 612.31 & 1176.26 & 989.53 & 842.05 \\
\hline 779.46 & 636.66 & 958.58 & 998.22 & 899.94 \\
\hline 782.10 & 713.91 & 1127.27 & 1046.92 & 963.25 \\
\hline 784.75 & 688.65 & 1063.25 & 1063.06 & 1032.62 \\
\hline 787.39 & 756.48 & 1045.36 & 1103.41 & 1111.70 \\
\hline 790.03 & 799.46 & 847.18 & 1101.89 & 1184.56 \\
\hline 792.67 & 873.77 & 1039.00 & 1180.19 & 1266.35 \\
\hline 795.31 & 945.68 & 1052.97 & 1213.89 & 1387.53 \\
\hline 797.95 & 1014.83 & 1098.68 & 1262.10 & 1507.93 \\
\hline 800.59 & 1126.02 & 984.43 & 1313.98 & 1619.81 \\
\hline 803.23 & 1247.01 & 1133.61 & 1407.51 & 1794.12 \\
\hline 805.86 & 1398.48 & 1115.73 & 1512.19 & 2010.92 \\
\hline 808.50 & 1590.30 & 1373.05 & 1667.20 & 2254.67 \\
\hline 811.13 & 1832.36 & 1578.36 & 1861.93 & 2577.47 \\
\hline 813.77 & 2046.70 & 1861.62 & 2065.55 & 3077.82 \\
\hline 816.40 & 2395.14 & 2055.87 & 2325.46 & 3702.95 \\
\hline 819.03 & 2874.72 & 2502.67 & 2669.72 & 4617.11 \\
\hline 821.66 & 3494.59 & 3315.33 & 3193.07 & 6000.58 \\
\hline 824.29 & 4475.89 & 4073.04 & 3916.78 & 7990.66 \\
\hline 826.92 & 6126.79 & 5107.45 & 5021.34 & 10943.76 \\
\hline 829.55 & 9156.68 & 6638.45 & 7016.13 & 14718.02 \\
\hline 832.17 & 15109.75 & 9390.40 & 11123.13 & 18560.36 \\
\hline 834.80 & 24996.37 & 14486.19 & 18987.06 & 21605.59 \\
\hline 837.42 & 37125.82 & 23405.72 & 31630.47 & 22861.40 \\
\hline 840.05 & 46568.26 & 34223.26 & 44295.57 & 22765.74 \\
\hline 842.67 & 49155.64 & 43581.25 & 53519.00 & 23412.91 \\
\hline 845.29 & 41097.66 & 49125.80 & 56263.60 & 27268.88 \\
\hline 847.92 & 26853.46 & 48295.47 & 47686.24 & 34662.08 \\
\hline 850.54 & 15447.40 & 38617.41 & 30232.63 & 43517.60 \\
\hline 853.15 & 9638.94 & 26516.64 & 17495.56 & 49143.50 \\
\hline 855.77 & 7409.41 & 17536.96 & 11893.79 & 47264.52 \\
\hline 858.39 & 6530.11 & 11981.16 & 9923.17 & 37912.19 \\
\hline 861.01 & 6013.90 & 9398.94 & 9342.04 & 26405.40 \\
\hline 863.62 & 5459.48 & 7937.91 & 8852.82 & 17737.72 \\
\hline
\end{tabular}




\section{Supplementary Information and Figures}

\begin{tabular}{|c|c|c|c|c|}
\hline 866.24 & 4743.39 & 7032.73 & 8016.63 & 12430.57 \\
\hline 868.85 & 4264.24 & 5736.32 & 6693.39 & 9346.93 \\
\hline 871.46 & 4010.88 & 4767.87 & 5341.40 & 7476.08 \\
\hline 874.08 & 4060.90 & 4136.15 & 4173.54 & 6390.79 \\
\hline 876.69 & 4235.71 & 3929.24 & 3337.63 & 5670.16 \\
\hline 879.30 & 4543.25 & 4050.97 & 2763.26 & 5188.17 \\
\hline 881.91 & 4826.28 & 4289.98 & 2434.99 & 4900.31 \\
\hline 884.51 & 5050.40 & 4632.81 & 2280.69 & 4739.05 \\
\hline 887.12 & 5271.84 & 5088.60 & 2289.19 & 4776.36 \\
\hline 889.73 & 5545.37 & 5441.99 & 2412.57 & 4850.29 \\
\hline 892.33 & 5784.09 & 5845.40 & 2779.41 & 5172.32 \\
\hline 894.94 & 5908.74 & 6490.29 & 3521.23 & 5685.11 \\
\hline 897.54 & 5465.98 & 6999.41 & 4792.00 & 6443.60 \\
\hline 900.14 & 4514.70 & 7348.33 & 6785.28 & 7362.96 \\
\hline 902.75 & 3322.89 & 7004.13 & 8994.03 & 8416.27 \\
\hline 905.35 & 2236.23 & 6229.47 & 10814.28 & 10060.22 \\
\hline 907.95 & 1509.00 & 4815.41 & 11293.43 & 12729.10 \\
\hline 910.55 & 1002.20 & 3186.78 & 10210.22 & 17207.84 \\
\hline 913.14 & 714.29 & 1886.82 & 7521.83 & 24711.07 \\
\hline 915.74 & 522.20 & 1202.91 & 4736.20 & 34818.00 \\
\hline 918.34 & 441.30 & 822.27 & 2893.65 & 43538.44 \\
\hline 920.93 & 391.33 & 621.49 & 1856.44 & 45794.72 \\
\hline 923.53 & 316.24 & 572.40 & 1297.69 & 39000.38 \\
\hline 926.12 & 307.04 & 541.93 & 964.39 & 26791.68 \\
\hline 928.71 & 251.41 & 474.58 & 764.41 & 16296.89 \\
\hline 931.30 & 268.17 & 509.79 & 628.71 & 10028.31 \\
\hline 933.89 & 215.41 & 402.35 & 515.99 & 6709.59 \\
\hline 936.48 & 236.38 & 386.59 & 454.23 & 4929.22 \\
\hline 939.07 & 218.43 & 427.10 & 399.61 & 3901.95 \\
\hline 941.66 & 176.96 & 356.85 & 343.48 & 3327.13 \\
\hline 944.25 & 156.42 & 345.04 & 308.83 & 3223.12 \\
\hline 946.83 & 156.16 & 399.21 & 320.23 & 3480.09 \\
\hline 949.42 & 108.93 & 319.31 & 253.48 & 4333.82 \\
\hline 952.00 & 138.09 & 375.51 & 239.95 & 5822.53 \\
\hline 954.58 & 142.12 & 251.87 & 209.00 & 7460.29 \\
\hline 957.17 & 109.12 & 246.72 & 195.26 & 8340.26 \\
\hline 959.75 & 124.31 & 242.27 & 190.01 & 7880.70 \\
\hline 962.33 & 143.33 & 237.98 & 165.27 & 6305.04 \\
\hline 964.91 & 132.33 & 289.65 & 172.35 & 4661.72 \\
\hline 967.49 & 143.45 & 243.63 & 148.78 & 3683.08 \\
\hline 970.06 & 155.36 & 221.76 & 136.55 & 3184.27 \\
\hline 972.64 & 118.59 & 160.02 & 124.05 & 2900.56 \\
\hline 975.22 & 144.05 & 366.27 & 164.99 & 2486.48 \\
\hline 977.79 & 136.24 & 217.00 & 116.04 & 1981.97 \\
\hline
\end{tabular}


Supplementary Information and Figures

\begin{tabular}{|c|c|c|c|c|}
\hline 980.36 & 160.31 & 333.07 & 125.04 & 1485.70 \\
\hline 982.94 & 186.90 & 387.28 & 142.06 & 1092.42 \\
\hline 985.51 & 175.62 & 268.40 & 116.80 & 849.56 \\
\hline 988.08 & 144.47 & 347.55 & 115.94 & 717.87 \\
\hline 990.65 & 131.15 & 328.50 & 115.64 & 559.16 \\
\hline 993.22 & 97.05 & 364.96 & 111.98 & 507.28 \\
\hline 995.79 & 99.88 & 248.47 & 82.11 & 435.53 \\
\hline 998.36 & 72.53 & 326.53 & 108.05 & 391.50 \\
\hline 1000.92 & 44.32 & 184.54 & 76.03 & 382.57 \\
\hline 1003.49 & 83.02 & 131.96 & 59.06 & 352.33 \\
\hline 1006.05 & 97.86 & 125.24 & 62.04 & 341.65 \\
\hline 1008.62 & 99.81 & 193.70 & 72.77 & 292.55 \\
\hline 1011.18 & 70.80 & 324.62 & 81.28 & 250.65 \\
\hline 1013.74 & 108.31 & 200.98 & 67.47 & 216.57 \\
\hline 1016.30 & 84.55 & 196.22 & 55.87 & 231.96 \\
\hline 1018.86 & 83.01 & 175.50 & 56.38 & 195.50 \\
\hline 1021.42 & 90.59 & 304.66 & 73.31 & 181.42 \\
\hline 1023.98 & 69.25 & 243.89 & 59.09 & 154.54 \\
\hline 1026.54 & 81.23 & 125.17 & 43.27 & 160.27 \\
\hline 1029.09 & 61.14 & 247.39 & 59.54 & 145.65 \\
\hline 1031.65 & 85.07 & 244.82 & 52.45 & 143.50 \\
\hline 1034.21 & 61.27 & 298.33 & 71.18 & 131.30 \\
\hline 1036.76 & 43.02 & 205.70 & 46.59 & 128.95 \\
\hline 1039.31 & 67.34 & 312.58 & 62.74 & 106.36 \\
\hline 1041.86 & 31.10 & 330.22 & 74.97 & 111.89 \\
\hline 1044.41 & 66.74 & 327.69 & 55.58 & 105.45 \\
\hline 1046.96 & 56.86 & 118.19 & 27.67 & 117.27 \\
\hline 1049.51 & 94.93 & 323.84 & 77.20 & 104.54 \\
\hline 1052.06 & 66.14 & 328.84 & 55.61 & 101.45 \\
\hline 1054.61 & 35.54 & 291.75 & 64.20 & 109.24 \\
\hline 1057.16 & 84.24 & 193.44 & 37.74 & 126.99 \\
\hline 1059.70 & 66.19 & 196.39 & 40.26 & 107.44 \\
\hline 1062.25 & 54.34 & 275.49 & 46.69 & 123.48 \\
\hline 1064.79 & 65.50 & 283.33 & 65.81 & 103.56 \\
\hline 1067.33 & 53.76 & 345.99 & 62.97 & 113.85 \\
\hline 1069.87 & 76.37 & 287.63 & 44.91 & 130.95 \\
\hline 1072.42 & 60.04 & 333.30 & 60.90 & 123.55 \\
\hline 1074.96 & 69.58 & 231.89 & 40.58 & 110.79 \\
\hline 1077.49 & 87.49 & 291.34 & 45.84 & 115.33 \\
\hline 1080.03 & 56.95 & 331.89 & 52.52 & 129.20 \\
\hline 1082.57 & 106.03 & 232.90 & 41.35 & 118.96 \\
\hline 1085.11 & 49.08 & 320.36 & 60.13 & 153.57 \\
\hline 1087.64 & 44.56 & 224.03 & 37.13 & 141.53 \\
\hline 1090.18 & 51.91 & 226.44 & 31.65 & 154.99 \\
\hline
\end{tabular}




\section{Supplementary Information and Figures}

\begin{tabular}{|c|c|c|c|c|}
\hline 1092.71 & 71.94 & 324.57 & 41.50 & 121.57 \\
\hline 1095.24 & 86.15 & 337.27 & 81.79 & 132.90 \\
\hline 1097.78 & 82.87 & 344.63 & 61.84 & 137.66 \\
\hline 1100.31 & 81.49 & 360.03 & 56.65 & 115.56 \\
\hline 1102.84 & 55.39 & 234.32 & 34.38 & 137.26 \\
\hline 1105.37 & 54.53 & 297.57 & 47.93 & 126.68 \\
\hline 1107.89 & 74.67 & 307.18 & 47.27 & 110.45 \\
\hline 1110.42 & 44.28 & 222.60 & 33.97 & 93.25 \\
\hline 1112.95 & 56.83 & 358.71 & 69.60 & 125.48 \\
\hline 1115.47 & 87.49 & 332.69 & 49.78 & 116.78 \\
\hline 1118.00 & 89.36 & 346.35 & 54.62 & 142.45 \\
\hline 1120.52 & 79.14 & 287.05 & 43.09 & 112.26 \\
\hline 1123.04 & 43.99 & 315.63 & 59.05 & 148.36 \\
\hline 1125.57 & 71.27 & 318.66 & 55.74 & 129.91 \\
\hline 1128.09 & 67.14 & 177.07 & 32.89 & 139.19 \\
\hline 1130.61 & 105.83 & 305.60 & 43.60 & 154.63 \\
\hline 1133.13 & 61.06 & 292.28 & 46.24 & 123.15 \\
\hline 1135.65 & 51.01 & 368.16 & 57.84 & 140.85 \\
\hline 1138.16 & 93.54 & 279.23 & 34.98 & 134.33 \\
\hline 1140.68 & 45.23 & 350.53 & 61.07 & 91.79 \\
\hline 1143.20 & 64.90 & 231.38 & 41.53 & 64.69 \\
\hline 1145.71 & 31.30 & 215.06 & 36.79 & 115.79 \\
\hline 1148.22 & 65.56 & 152.73 & 19.35 & 115.59 \\
\hline 1150.74 & 43.83 & 291.07 & 46.31 & 121.27 \\
\hline 1153.25 & 60.98 & 304.07 & 47.63 & 141.31 \\
\hline 1155.76 & 62.89 & 309.81 & 68.62 & 147.47 \\
\hline 1158.27 & 67.71 & 220.66 & 33.27 & 143.55 \\
\hline 1160.78 & 63.85 & 328.26 & 57.50 & 123.57 \\
\hline 1163.29 & 37.84 & 346.03 & 67.22 & 98.68 \\
\hline 1165.80 & 69.19 & 311.07 & 69.65 & 153.31 \\
\hline 1168.30 & 106.01 & 305.85 & 59.88 & 124.63 \\
\hline 1170.81 & 54.82 & 148.57 & 35.38 & 117.96 \\
\hline 1173.31 & 51.00 & 323.94 & 76.58 & 92.38 \\
\hline 1175.82 & 49.32 & 322.46 & 58.49 & 109.71 \\
\hline 1178.32 & 51.15 & 208.58 & 40.33 & 95.26 \\
\hline 1180.82 & 70.27 & 278.09 & 53.98 & 133.45 \\
\hline 1183.32 & 71.10 & 315.41 & 65.41 & 121.48 \\
\hline 1185.82 & 78.87 & 322.02 & 106.80 & 115.36 \\
\hline 1188.32 & 79.29 & 133.52 & 29.97 & 95.45 \\
\hline 1190.82 & 92.45 & 147.24 & 35.68 & 127.49 \\
\hline 1193.32 & 70.01 & 134.01 & 33.56 & 87.00 \\
\hline 1195.82 & 15.00 & 319.55 & 69.55 & 95.07 \\
\hline 1198.31 & 56.74 & 200.77 & 40.85 & 108.05 \\
\hline 1200.81 & 36.77 & 113.60 & 22.59 & 136.22 \\
\hline
\end{tabular}


Supplementary Information and Figures

\begin{tabular}{|c|c|c|c|c|}
\hline 1203.30 & 73.93 & 322.76 & 74.04 & 94.50 \\
\hline 1205.79 & 73.29 & 183.69 & 41.36 & 151.22 \\
\hline 1208.29 & 85.81 & 262.23 & 53.52 & 94.39 \\
\hline 1210.78 & 87.33 & 194.86 & 50.00 & 127.54 \\
\hline 1213.27 & 64.18 & 115.70 & 39.48 & 133.69 \\
\hline 1215.76 & 65.06 & 293.81 & 79.16 & 109.69 \\
\hline 1218.25 & 39.37 & 226.62 & 63.09 & 131.84 \\
\hline 1220.74 & 98.75 & 246.25 & 84.46 & 100.25 \\
\hline 1223.22 & 66.62 & 144.91 & 50.90 & 125.71 \\
\hline 1225.71 & 60.67 & 207.93 & 65.31 & 121.76 \\
\hline 1228.19 & 84.25 & 258.10 & 71.80 & 130.76 \\
\hline 1230.68 & 57.02 & 328.38 & 81.12 & 122.47 \\
\hline 1233.16 & 86.53 & 109.31 & 38.52 & 110.96 \\
\hline 1235.64 & 67.04 & 228.40 & 68.03 & 130.37 \\
\hline 1238.13 & 48.59 & 178.66 & 56.11 & 131.22 \\
\hline 1240.61 & 60.69 & 110.77 & 41.39 & 105.14 \\
\hline 1243.09 & 78.10 & 103.33 & 39.45 & 111.88 \\
\hline 1245.56 & 79.98 & 204.28 & 58.54 & 116.51 \\
\hline 1248.04 & 31.95 & 251.04 & 63.07 & 101.85 \\
\hline 1250.52 & 39.34 & 295.90 & 68.54 & 92.68 \\
\hline 1253.00 & 89.20 & 293.78 & 65.45 & 125.08 \\
\hline 1255.47 & 59.48 & 165.30 & 43.24 & 132.98 \\
\hline 1257.95 & 31.48 & 280.09 & 54.12 & 127.59 \\
\hline 1260.42 & 51.17 & 241.23 & 59.51 & 124.78 \\
\hline 1262.89 & 98.38 & 102.81 & 32.57 & 99.74 \\
\hline 1265.36 & 52.93 & 189.92 & 47.27 & 123.27 \\
\hline 1267.84 & 57.90 & 253.90 & 80.57 & 100.07 \\
\hline 1270.31 & 89.53 & 237.37 & 50.38 & 91.96 \\
\hline 1272.77 & 72.10 & 236.09 & 51.95 & 82.89 \\
\hline 1275.24 & 44.71 & 167.91 & 43.66 & 83.68 \\
\hline 1277.71 & 66.47 & 228.14 & 54.08 & 102.22 \\
\hline 1280.18 & 62.73 & 169.75 & 43.26 & 147.07 \\
\hline 1282.64 & 70.10 & 219.82 & 52.29 & 108.51 \\
\hline 1285.11 & 81.18 & 212.63 & 44.14 & 114.58 \\
\hline 1287.57 & 46.07 & 316.68 & 61.44 & 133.66 \\
\hline 1290.03 & 74.53 & 287.47 & 59.87 & 150.06 \\
\hline 1292.50 & 77.45 & 247.62 & 48.13 & 135.56 \\
\hline 1294.96 & 71.64 & 307.52 & 69.20 & 105.57 \\
\hline 1297.42 & 55.81 & 262.30 & 51.17 & 143.28 \\
\hline 1299.88 & 89.76 & 171.77 & 50.71 & 139.59 \\
\hline 1302.34 & 38.53 & 239.68 & 49.08 & 133.16 \\
\hline 1304.79 & 39.85 & 263.48 & 45.32 & 137.48 \\
\hline 1307.25 & 76.74 & 181.25 & 44.90 & 116.17 \\
\hline 1309.71 & 39.48 & 258.79 & 48.61 & 104.95 \\
\hline
\end{tabular}


Supplementary Information and Figures

\begin{tabular}{|c|c|c|c|c|}
\hline 1312.16 & 75.06 & 245.59 & 52.62 & 131.77 \\
\hline 1314.62 & 68.34 & 227.01 & 52.30 & 93.59 \\
\hline 1317.07 & 81.06 & 297.45 & 82.28 & 116.45 \\
\hline 1319.52 & 37.99 & 166.68 & 41.10 & 122.92 \\
\hline 1321.97 & 72.32 & 237.54 & 51.28 & 159.42 \\
\hline 1324.42 & 72.10 & 165.15 & 42.96 & 171.30 \\
\hline 1326.87 & 42.07 & 297.79 & 64.78 & 146.97 \\
\hline 1329.32 & 81.43 & 223.23 & 53.66 & 136.46 \\
\hline 1331.77 & 51.48 & 163.59 & 44.27 & 118.75 \\
\hline 1334.22 & 69.20 & 213.59 & 48.99 & 125.43 \\
\hline 1336.66 & 33.61 & 219.83 & 49.04 & 148.47 \\
\hline 1339.11 & 70.08 & 267.97 & 64.99 & 126.58 \\
\hline 1341.55 & 46.37 & 259.59 & 60.30 & 151.12 \\
\hline 1344.00 & 57.17 & 64.91 & 28.68 & 155.20 \\
\hline 1346.44 & 53.43 & 306.29 & 75.46 & 128.60 \\
\hline 1348.88 & 66.64 & 131.36 & 43.17 & 159.20 \\
\hline 1351.32 & 56.71 & 204.94 & 47.45 & 144.03 \\
\hline 1353.76 & 48.81 & 74.06 & 32.95 & 125.82 \\
\hline 1356.20 & 38.86 & 139.61 & 45.50 & 135.94 \\
\hline 1358.64 & 62.79 & 241.07 & 58.57 & 125.58 \\
\hline 1361.08 & 38.61 & 126.81 & 35.28 & 140.05 \\
\hline 1363.51 & 40.94 & 189.58 & 54.75 & 143.12 \\
\hline 1365.95 & 76.16 & 268.03 & 61.82 & 131.27 \\
\hline 1368.39 & 32.14 & 262.24 & 53.59 & 114.89 \\
\hline 1370.82 & 74.68 & 57.58 & 33.86 & 135.94 \\
\hline 1373.25 & 58.33 & 254.52 & 74.00 & 118.98 \\
\hline 1375.68 & 99.34 & 103.46 & 44.57 & 109.59 \\
\hline 1378.12 & 24.60 & 195.52 & 43.07 & 144.32 \\
\hline 1380.55 & 52.01 & 273.54 & 70.57 & 140.08 \\
\hline 1382.98 & 75.70 & 133.98 & 44.10 & 164.61 \\
\hline 1385.40 & 79.24 & 211.02 & 48.10 & 147.73 \\
\hline 1387.83 & 57.45 & 146.67 & 37.13 & 148.09 \\
\hline 1390.26 & 52.05 & 221.70 & 57.33 & 185.54 \\
\hline 1392.69 & 71.07 & 278.84 & 62.53 & 159.00 \\
\hline 1395.11 & 24.31 & 91.84 & 28.06 & 157.87 \\
\hline 1397.54 & 73.52 & 281.53 & 61.42 & 154.31 \\
\hline 1399.96 & 58.54 & 184.77 & 41.75 & 145.00 \\
\hline 1402.38 & 48.77 & 281.52 & 75.52 & 155.99 \\
\hline 1404.80 & 60.00 & 169.88 & 52.91 & 126.99 \\
\hline 1407.22 & 63.93 & 277.76 & 60.71 & 138.42 \\
\hline 1409.64 & 76.46 & 238.05 & 56.67 & 143.10 \\
\hline 1412.06 & 106.85 & 332.47 & 66.43 & 127.75 \\
\hline 1414.48 & 98.68 & 299.72 & 67.93 & 121.84 \\
\hline 1416.90 & 97.27 & 204.52 & 55.55 & 147.40 \\
\hline
\end{tabular}




\section{Supplementary Information and Figures}

\begin{tabular}{|c|c|c|c|c|}
\hline 1419.32 & 93.07 & 302.67 & 77.21 & 127.13 \\
\hline 1421.73 & 93.13 & 323.41 & 103.47 & 116.50 \\
\hline 1424.15 & 102.59 & 206.09 & 63.07 & 135.46 \\
\hline 1426.56 & 86.76 & 252.96 & 58.49 & 124.64 \\
\hline 1428.97 & 99.00 & 282.78 & 86.62 & 121.73 \\
\hline 1431.39 & 46.85 & 211.37 & 59.71 & 150.04 \\
\hline 1433.80 & 65.86 & 226.27 & 63.34 & 69.27 \\
\hline 1436.21 & 107.69 & 276.94 & 73.63 & 113.19 \\
\hline 1438.62 & 65.28 & 336.62 & 127.69 & 121.65 \\
\hline 1441.03 & 76.20 & 334.94 & 82.99 & 91.22 \\
\hline 1443.43 & 47.83 & 223.14 & 74.35 & 147.85 \\
\hline 1445.84 & 82.13 & 343.02 & 101.64 & 128.12 \\
\hline 1448.25 & 63.76 & 334.25 & 114.13 & 109.23 \\
\hline 1450.65 & 56.11 & 342.73 & 122.29 & 84.37 \\
\hline 1453.06 & 51.74 & 303.04 & 134.85 & 76.79 \\
\hline 1455.46 & 69.69 & 230.36 & 115.58 & 114.82 \\
\hline 1457.86 & 106.77 & 264.79 & 127.64 & 131.13 \\
\hline 1460.27 & 78.93 & 230.46 & 127.20 & 114.65 \\
\hline 1462.67 & 82.32 & 293.56 & 131.82 & 122.90 \\
\hline 1465.07 & 87.94 & 316.02 & 145.18 & 147.81 \\
\hline 1467.47 & 75.49 & 326.93 & 141.64 & 119.90 \\
\hline 1469.86 & 62.73 & 220.12 & 122.61 & 118.30 \\
\hline 1472.26 & 69.41 & 359.71 & 132.57 & 138.97 \\
\hline 1474.66 & 80.47 & 162.81 & 107.73 & 140.05 \\
\hline 1477.05 & 59.22 & 234.13 & 116.06 & 75.91 \\
\hline 1479.45 & 91.01 & 343.92 & 153.69 & 122.17 \\
\hline 1481.84 & 82.29 & 360.40 & 151.85 & 142.96 \\
\hline 1484.24 & 87.11 & 286.98 & 141.45 & 147.61 \\
\hline 1486.63 & 71.07 & 322.11 & 158.01 & 102.13 \\
\hline 1489.02 & 37.79 & 237.42 & 142.24 & 120.58 \\
\hline 1491.41 & 72.54 & 320.41 & 150.27 & 92.39 \\
\hline 1493.80 & 47.60 & 358.21 & 159.07 & 129.52 \\
\hline 1496.19 & 67.55 & 254.64 & 118.93 & 111.75 \\
\hline 1498.58 & 95.10 & 299.22 & 120.97 & 99.55 \\
\hline 1500.96 & 43.81 & 240.60 & 98.77 & 139.57 \\
\hline 1503.35 & 65.62 & 317.98 & 97.00 & 118.16 \\
\hline 1505.74 & 64.03 & 371.24 & 98.30 & 142.34 \\
\hline 1508.12 & 36.99 & 331.80 & 91.67 & 102.96 \\
\hline 1510.50 & 31.47 & 241.36 & 72.77 & 99.92 \\
\hline 1512.89 & 75.95 & 298.04 & 75.47 & 98.30 \\
\hline 1515.27 & 81.59 & 326.72 & 78.04 & 175.14 \\
\hline 1517.65 & 62.99 & 246.69 & 64.65 & 136.70 \\
\hline 1520.03 & 55.40 & 338.35 & 75.19 & 161.71 \\
\hline 1522.41 & 78.67 & 366.09 & 95.76 & 130.19 \\
\hline
\end{tabular}




\section{Supplementary Information and Figures}

\begin{tabular}{|c|c|c|c|c|}
\hline 1524.79 & 54.36 & 305.75 & 70.90 & 103.77 \\
\hline 1527.17 & 76.56 & 182.67 & 44.74 & 134.77 \\
\hline 1529.54 & 82.03 & 379.19 & 91.88 & 116.29 \\
\hline 1531.92 & 50.27 & 159.54 & 47.92 & 121.36 \\
\hline 1534.30 & 53.82 & 236.74 & 58.00 & 125.23 \\
\hline 1536.67 & 31.50 & 356.38 & 70.61 & 134.64 \\
\hline 1539.04 & 71.15 & 139.81 & 29.89 & 142.40 \\
\hline 1541.42 & 49.07 & 218.57 & 40.90 & 148.71 \\
\hline 1543.79 & 31.96 & 149.05 & 38.25 & 150.39 \\
\hline 1546.16 & 12.71 & 129.76 & 30.29 & 120.00 \\
\hline 1548.53 & 62.44 & 305.90 & 59.34 & 141.82 \\
\hline 1550.90 & 76.09 & 350.22 & 73.02 & 187.02 \\
\hline 1553.27 & 67.76 & 339.91 & 68.64 & 158.81 \\
\hline 1555.63 & 93.80 & 222.23 & 45.96 & 167.90 \\
\hline 1558.00 & 73.86 & 228.09 & 47.98 & 129.71 \\
\hline 1560.37 & 112.14 & 203.21 & 45.43 & 141.72 \\
\hline 1562.73 & 68.23 & 340.98 & 54.49 & 128.11 \\
\hline 1565.10 & 44.95 & 236.81 & 48.21 & 143.49 \\
\hline 1567.46 & 63.93 & 292.37 & 58.30 & 130.52 \\
\hline 1569.82 & 61.23 & 126.91 & 40.53 & 163.21 \\
\hline 1572.18 & 62.60 & 199.42 & 53.82 & 156.75 \\
\hline 1574.54 & 45.34 & 199.73 & 51.55 & 167.77 \\
\hline 1576.90 & 74.09 & 212.07 & 48.11 & 142.52 \\
\hline 1579.26 & 64.17 & 341.92 & 90.53 & 162.18 \\
\hline 1581.62 & 26.86 & 292.50 & 84.63 & 163.44 \\
\hline 1583.98 & 69.11 & 338.70 & 70.89 & 141.79 \\
\hline 1586.33 & 83.53 & 293.64 & 68.82 & 147.22 \\
\hline 1588.69 & 4.47 & 328.60 & 77.82 & 127.47 \\
\hline 1591.04 & 72.43 & 148.12 & 42.89 & 110.58 \\
\hline 1593.40 & 78.90 & 328.63 & 76.72 & 110.49 \\
\hline 1595.75 & 112.19 & 209.26 & 53.99 & 125.37 \\
\hline 1598.10 & 68.84 & 233.82 & 59.59 & 145.23 \\
\hline 1600.45 & 95.16 & 307.03 & 69.79 & 126.50 \\
\hline 1602.81 & 78.84 & 211.07 & 61.26 & 129.10 \\
\hline 1605.15 & 74.14 & 344.07 & 83.00 & 139.60 \\
\hline 1607.50 & 91.85 & 320.08 & 71.53 & 152.60 \\
\hline 1609.85 & 61.78 & 213.55 & 62.70 & 151.06 \\
\hline 1612.20 & 102.02 & 381.95 & 110.27 & 117.93 \\
\hline 1614.54 & 64.18 & 233.06 & 74.78 & 139.05 \\
\hline 1616.89 & 106.25 & 352.52 & 93.82 & 102.57 \\
\hline 1619.23 & 120.48 & 380.83 & 117.25 & 155.87 \\
\hline 1621.58 & 105.88 & 350.57 & 89.43 & 109.99 \\
\hline 1623.92 & 104.29 & 218.97 & 74.73 & 139.21 \\
\hline 1626.26 & 121.81 & 163.85 & 62.57 & 132.24 \\
\hline
\end{tabular}




\section{Supplementary Information and Figures}

\begin{tabular}{|c|c|c|c|c|}
\hline 1628.60 & 132.65 & 239.60 & 78.18 & 130.87 \\
\hline 1630.95 & 132.75 & 317.08 & 88.85 & 173.12 \\
\hline 1633.28 & 115.91 & 294.80 & 90.23 & 128.38 \\
\hline 1635.62 & 144.48 & 219.01 & 80.23 & 125.93 \\
\hline 1637.96 & 139.46 & 303.20 & 98.11 & 82.63 \\
\hline 1640.30 & 134.76 & 370.98 & 110.05 & 105.55 \\
\hline 1642.63 & 189.12 & 374.73 & 132.72 & 122.76 \\
\hline 1644.97 & 183.09 & 408.59 & 139.83 & 136.66 \\
\hline 1647.30 & 194.92 & 285.38 & 119.14 & 105.20 \\
\hline 1649.64 & 207.41 & 409.57 & 152.24 & 126.91 \\
\hline 1651.97 & 255.37 & 426.83 & 183.62 & 161.95 \\
\hline 1654.30 & 281.68 & 448.54 & 126.69 & 126.88 \\
\hline 1656.63 & 358.05 & 679.59 & 170.00 & 122.85 \\
\hline 1658.96 & 389.19 & 602.21 & 135.87 & 140.13 \\
\hline 1661.29 & 392.53 & 570.71 & 134.28 & 104.57 \\
\hline 1663.62 & 362.39 & 500.19 & 156.15 & 87.86 \\
\hline 1665.95 & 335.85 & 532.19 & 169.36 & 106.08 \\
\hline 1668.28 & 361.97 & 493.31 & 172.20 & 87.80 \\
\hline 1670.60 & 295.15 & 479.37 & 190.77 & 87.87 \\
\hline 1672.93 & 300.64 & 495.38 & 169.85 & 89.75 \\
\hline 1675.25 & 269.69 & 346.03 & 148.25 & 122.39 \\
\hline 1677.58 & 268.70 & 404.67 & 166.66 & 104.15 \\
\hline 1679.90 & 250.47 & 347.52 & 150.89 & 90.90 \\
\hline 1682.22 & 298.34 & 492.60 & 177.93 & 78.82 \\
\hline 1684.54 & 316.32 & 481.51 & 207.37 & 108.84 \\
\hline 1686.86 & 284.56 & 553.80 & 191.61 & 87.01 \\
\hline 1689.18 & 259.46 & 533.37 & 190.51 & 105.73 \\
\hline 1691.50 & 248.92 & 368.12 & 137.88 & 113.56 \\
\hline 1693.82 & 258.38 & 405.85 & 137.35 & 86.16 \\
\hline 1696.13 & 189.95 & 506.89 & 140.78 & 129.04 \\
\hline 1698.45 & 179.03 & 317.79 & 101.62 & 149.39 \\
\hline 1700.77 & 185.51 & 311.06 & 101.08 & 151.98 \\
\hline 1703.08 & 192.35 & 387.00 & 117.95 & 96.58 \\
\hline 1705.39 & 141.59 & 398.51 & 110.35 & 85.57 \\
\hline 1707.71 & 184.99 & 327.50 & 88.81 & 122.46 \\
\hline 1710.02 & 170.10 & 382.99 & 109.01 & 95.92 \\
\hline 1712.33 & 180.86 & 337.98 & 81.07 & 85.10 \\
\hline 1714.64 & 212.53 & 288.32 & 63.04 & 92.41 \\
\hline 1716.95 & 188.20 & 449.48 & 90.13 & 91.01 \\
\hline 1719.26 & 127.28 & 347.39 & 66.93 & 97.29 \\
\hline 1721.56 & 120.66 & 475.92 & 119.29 & 77.07 \\
\hline 1723.87 & 131.25 & 484.44 & 72.78 & 79.75 \\
\hline 1726.18 & 122.49 & 458.52 & 88.34 & 69.19 \\
\hline 1728.48 & 100.54 & 348.64 & 68.09 & 96.15 \\
\hline
\end{tabular}


Supplementary Information and Figures

\begin{tabular}{|c|c|c|c|c|}
\hline 1730.79 & 115.19 & 271.26 & 47.72 & 65.08 \\
\hline 1733.09 & 123.69 & 310.45 & 79.81 & 117.79 \\
\hline 1735.39 & 76.40 & 290.75 & 64.43 & 90.28 \\
\hline 1737.69 & 67.51 & 315.63 & 67.15 & 91.21 \\
\hline 1740.00 & 69.33 & 214.98 & 53.83 & 114.59 \\
\hline 1742.30 & 70.24 & 211.32 & 57.52 & 96.93 \\
\hline 1744.59 & 44.51 & 324.89 & 91.10 & 121.43 \\
\hline 1746.89 & 72.48 & 171.63 & 45.35 & 81.04 \\
\hline 1749.19 & 101.31 & 305.28 & 72.40 & 133.98 \\
\hline 1751.49 & 56.59 & 323.02 & 83.18 & 84.74 \\
\hline 1753.78 & 105.32 & 289.15 & 66.66 & 123.04 \\
\hline 1756.08 & 67.25 & 170.84 & 35.80 & 111.93 \\
\hline 1758.37 & 82.68 & 312.96 & 65.88 & 132.47 \\
\hline 1760.67 & 73.22 & 192.44 & 42.98 & 135.72 \\
\hline 1762.96 & 77.96 & 181.81 & 34.36 & 131.77 \\
\hline 1765.25 & 63.29 & 269.96 & 70.73 & 88.59 \\
\hline 1767.54 & 54.23 & 175.39 & 45.37 & 97.89 \\
\hline 1769.83 & 88.02 & 276.67 & 64.80 & 107.11 \\
\hline 1772.12 & 60.66 & 227.74 & 42.60 & 113.67 \\
\hline 1774.41 & 66.50 & 245.74 & 40.57 & 131.57 \\
\hline 1776.70 & 47.25 & 306.50 & 58.44 & 131.27 \\
\hline 1778.99 & 71.14 & 192.46 & 29.06 & 153.43 \\
\hline 1781.27 & 52.18 & 291.18 & 77.73 & 182.13 \\
\hline 1783.56 & 70.27 & 182.40 & 32.59 & 112.51 \\
\hline 1785.84 & 60.86 & 330.91 & 54.01 & 112.27 \\
\hline 1788.12 & 76.15 & 273.73 & 37.40 & 103.65 \\
\hline 1790.41 & 31.74 & 297.12 & 61.59 & 137.44 \\
\hline 1792.69 & 68.93 & 309.08 & 52.45 & 135.39 \\
\hline 1794.97 & 29.87 & 327.14 & 64.20 & 147.68 \\
\hline 1797.25 & 61.75 & 189.18 & 26.59 & 143.54 \\
\hline 1799.53 & 74.89 & 189.05 & 31.77 & 128.55 \\
\hline 1801.81 & 35.32 & 344.29 & 60.69 & 146.29 \\
\hline 1804.09 & 110.05 & 276.68 & 41.64 & 144.22 \\
\hline 1806.36 & 64.28 & 187.61 & 23.02 & 135.69 \\
\hline 1808.64 & 82.06 & 283.95 & 53.73 & 127.79 \\
\hline 1810.92 & 51.99 & 248.75 & 34.78 & 142.07 \\
\hline 1813.19 & 44.86 & 273.10 & 75.32 & 143.81 \\
\hline 1815.46 & 55.14 & 314.87 & 40.94 & 130.48 \\
\hline 1817.74 & 66.31 & 250.78 & 37.68 & 160.23 \\
\hline 1820.01 & 85.28 & 298.00 & 45.42 & 158.08 \\
\hline 1822.28 & 39.66 & 259.57 & 66.23 & 179.83 \\
\hline 1824.55 & 75.13 & 234.46 & 36.84 & 188.30 \\
\hline 1826.82 & 50.41 & 292.26 & 81.62 & 162.78 \\
\hline 1829.09 & 71.28 & 295.45 & 47.92 & 155.19 \\
\hline
\end{tabular}


Supplementary Information and Figures

\begin{tabular}{|c|c|c|c|c|}
\hline 1831.36 & 49.16 & 315.80 & 47.30 & 105.25 \\
\hline 1833.62 & 36.64 & 276.12 & 66.97 & 150.42 \\
\hline 1835.89 & 91.22 & 245.19 & 35.34 & 117.47 \\
\hline 1838.16 & 73.05 & 165.07 & 25.28 & 144.45 \\
\hline 1840.42 & 32.99 & 208.84 & 30.76 & 158.06 \\
\hline 1842.68 & 53.72 & 255.82 & 51.76 & 154.92 \\
\hline 1844.95 & 70.56 & 298.24 & 69.93 & 161.10 \\
\hline 1847.21 & 45.81 & 245.69 & 43.21 & 130.25 \\
\hline 1849.47 & 55.00 & 210.46 & 35.71 & 146.96 \\
\hline 1851.73 & 72.20 & 281.89 & 50.25 & 160.71 \\
\hline 1853.99 & 51.85 & 262.58 & 48.62 & 186.48 \\
\hline 1856.25 & 28.66 & 266.33 & 43.96 & 127.64 \\
\hline 1858.51 & 96.02 & 259.35 & 43.94 & 154.37 \\
\hline 1860.77 & 26.54 & 290.46 & 39.24 & 150.40 \\
\hline 1863.02 & 62.11 & 252.81 & 37.55 & 154.79 \\
\hline 1865.28 & 45.63 & 263.91 & 61.02 & 135.17 \\
\hline 1867.53 & 42.26 & 272.56 & 38.23 & 135.19 \\
\hline 1869.79 & 29.15 & 196.95 & 32.84 & 124.30 \\
\hline 1872.04 & 56.84 & 191.95 & 29.22 & 161.40 \\
\hline 1874.29 & 91.24 & 248.42 & 38.36 & 100.60 \\
\hline 1876.54 & 14.10 & 294.93 & 45.57 & 100.88 \\
\hline 1878.79 & 65.81 & 119.04 & 29.51 & 88.97 \\
\hline 1881.04 & 79.73 & 47.47 & 12.59 & 115.34 \\
\hline 1883.29 & 46.80 & 142.11 & 19.90 & 148.00 \\
\hline 1885.54 & 60.94 & 146.75 & 29.55 & 127.03 \\
\hline 1887.79 & 64.18 & 186.29 & 37.83 & 111.39 \\
\hline 1890.04 & 73.63 & 56.87 & 21.17 & 155.37 \\
\hline 1892.28 & 76.18 & 230.31 & 37.02 & 121.93 \\
\hline 1894.53 & 47.30 & 265.60 & 61.77 & 146.38 \\
\hline 1896.77 & 83.32 & 253.59 & 47.92 & 106.97 \\
\hline 1899.01 & 49.40 & 179.44 & 33.41 & 71.99 \\
\hline 1901.26 & 57.75 & 211.58 & 52.23 & 110.73 \\
\hline 1903.50 & 88.80 & 195.39 & 32.55 & 85.01 \\
\hline 1905.74 & 12.06 & 268.10 & 57.24 & 140.04 \\
\hline 1907.98 & 21.28 & 279.80 & 57.03 & 130.17 \\
\hline 1910.22 & 61.81 & 202.21 & 39.65 & 143.12 \\
\hline 1912.46 & 56.55 & 254.45 & 64.88 & 111.96 \\
\hline 1914.70 & 60.00 & 266.60 & 59.51 & 118.23 \\
\hline 1916.93 & 88.86 & 276.38 & 56.87 & 122.26 \\
\hline 1919.17 & 72.53 & 290.40 & 59.83 & 85.92 \\
\hline 1921.40 & 40.06 & 247.78 & 60.46 & 103.24 \\
\hline 1923.64 & 105.19 & 226.40 & 31.76 & 96.93 \\
\hline 1925.87 & 68.02 & 219.73 & 34.83 & 75.70 \\
\hline 1928.11 & 56.00 & 279.16 & 47.54 & 114.94 \\
\hline
\end{tabular}


Supplementary Information and Figures

\begin{tabular}{|c|c|c|c|c|}
\hline 1930.34 & 37.03 & 207.50 & 40.91 & 105.07 \\
\hline 1932.57 & 72.95 & 283.76 & 73.58 & 115.39 \\
\hline 1934.80 & 87.26 & 166.80 & 25.33 & 106.08 \\
\hline 1937.03 & 37.96 & 295.72 & 64.04 & 116.67 \\
\hline 1939.26 & 56.35 & 155.53 & 31.18 & 112.63 \\
\hline 1941.49 & 75.73 & 284.72 & 46.87 & 101.95 \\
\hline 1943.71 & 84.93 & 307.36 & 77.88 & 131.46 \\
\hline 1945.94 & 61.77 & 304.04 & 66.73 & 93.91 \\
\hline 1948.17 & 57.83 & 313.00 & 48.56 & 145.40 \\
\hline 1950.39 & 89.57 & 284.59 & 56.85 & 136.50 \\
\hline 1952.61 & 78.68 & 302.09 & 64.34 & 131.05 \\
\hline 1954.84 & 95.27 & 331.54 & 61.63 & 104.90 \\
\hline 1957.06 & 103.37 & 267.12 & 45.26 & 77.19 \\
\hline 1959.28 & 51.54 & 310.84 & 46.43 & 87.86 \\
\hline 1961.50 & 71.57 & 285.84 & 60.50 & 133.07 \\
\hline 1963.72 & 61.36 & 306.60 & 52.04 & 109.64 \\
\hline 1965.94 & 118.56 & 218.33 & 34.91 & 119.61 \\
\hline 1968.16 & 61.37 & 118.56 & 26.05 & 152.68 \\
\hline 1970.38 & 78.99 & 300.86 & 68.40 & 125.62 \\
\hline 1972.59 & 56.65 & 221.86 & 40.58 & 129.24 \\
\hline 1974.81 & 56.97 & 275.40 & 53.89 & 159.52 \\
\hline 1977.02 & 65.24 & 304.55 & 60.41 & 99.10 \\
\hline 1979.24 & 48.50 & 326.50 & 80.20 & 127.12 \\
\hline 1981.45 & 65.25 & 163.78 & 34.32 & 92.22 \\
\hline 1983.67 & 77.70 & 221.49 & 43.68 & 101.95 \\
\hline 1985.88 & 70.88 & 265.47 & 64.67 & 109.64 \\
\hline 1988.09 & 94.25 & 257.24 & 71.70 & 91.27 \\
\hline 1990.30 & 72.80 & 244.39 & 43.94 & 106.80 \\
\hline 1992.51 & 86.99 & 287.19 & 52.74 & 100.16 \\
\hline 1994.72 & 54.26 & 227.13 & 50.97 & 91.93 \\
\hline 1996.92 & 46.50 & 169.03 & 37.51 & 88.66 \\
\hline 1999.13 & 59.72 & 226.81 & 48.92 & 98.61 \\
\hline 2001.34 & 68.62 & 309.70 & 61.50 & 88.09 \\
\hline 2003.54 & 79.53 & 304.11 & 71.88 & 103.34 \\
\hline 2005.75 & 86.52 & 274.74 & 66.16 & 71.28 \\
\hline 2007.95 & 69.93 & 123.20 & 24.44 & 116.16 \\
\hline 2010.16 & 108.31 & 122.06 & 33.49 & 120.96 \\
\hline 2012.36 & 25.09 & 124.38 & 34.38 & 96.99 \\
\hline 2014.56 & 74.04 & 271.39 & 49.90 & 145.40 \\
\hline 2016.76 & 84.44 & 207.11 & 50.96 & 139.23 \\
\hline 2018.96 & 76.48 & 136.11 & 35.25 & 128.53 \\
\hline 2021.16 & 54.07 & 207.34 & 47.38 & 128.02 \\
\hline 2023.36 & 83.26 & 218.84 & 50.05 & 107.26 \\
\hline 2025.56 & 104.20 & 341.64 & 55.48 & 139.29 \\
\hline
\end{tabular}


Supplementary Information and Figures

\begin{tabular}{|c|c|c|c|c|}
\hline 2027.75 & 65.51 & 272.58 & 56.88 & 142.02 \\
\hline 2029.95 & 92.72 & 274.88 & 57.68 & 135.78 \\
\hline 2032.14 & 72.70 & 287.76 & 53.59 & 162.68 \\
\hline 2034.34 & 74.75 & 270.91 & 65.86 & 115.64 \\
\hline 2036.53 & 80.03 & 338.60 & 83.30 & 135.41 \\
\hline 2038.72 & 63.78 & 349.38 & 65.82 & 117.97 \\
\hline 2040.92 & 70.61 & 314.89 & 79.60 & 120.00 \\
\hline 2043.11 & 74.93 & 355.91 & 73.18 & 168.38 \\
\hline 2045.30 & 79.58 & 143.53 & 38.96 & 198.27 \\
\hline 2047.49 & 57.33 & 275.95 & 58.48 & 116.63 \\
\hline 2049.68 & 38.52 & 284.70 & 57.47 & 144.61 \\
\hline 2051.86 & 66.91 & 320.48 & 76.56 & 137.32 \\
\hline 2054.05 & 63.56 & 293.11 & 64.91 & 117.13 \\
\hline 2056.24 & 70.50 & 203.16 & 57.21 & 122.25 \\
\hline 2058.42 & 72.12 & 130.10 & 45.00 & 135.15 \\
\hline 2060.61 & 98.04 & 247.56 & 56.03 & 179.59 \\
\hline 2062.79 & 75.18 & 153.27 & 38.16 & 141.28 \\
\hline 2064.97 & 58.24 & 201.72 & 44.46 & 128.40 \\
\hline 2067.16 & 97.57 & 330.50 & 69.60 & 135.16 \\
\hline 2069.34 & 48.99 & 327.69 & 81.96 & 141.81 \\
\hline 2071.52 & 79.23 & 284.99 & 58.30 & 130.25 \\
\hline 2073.70 & 43.71 & 211.57 & 51.41 & 122.56 \\
\hline 2075.88 & 43.46 & 347.37 & 79.62 & 131.37 \\
\hline 2078.06 & 72.39 & 266.54 & 55.76 & 124.76 \\
\hline 2080.23 & 65.26 & 323.55 & 80.74 & 149.36 \\
\hline 2082.41 & 82.05 & 269.66 & 61.09 & 129.56 \\
\hline 2084.59 & 75.22 & 336.20 & 74.13 & 143.15 \\
\hline 2086.76 & 59.23 & 215.41 & 41.12 & 142.67 \\
\hline 2088.94 & 58.10 & 201.76 & 44.10 & 118.72 \\
\hline 2091.11 & 78.06 & 324.87 & 66.99 & 98.27 \\
\hline 2093.28 & 78.40 & 332.29 & 72.18 & 121.13 \\
\hline 2095.46 & 55.32 & 331.64 & 94.50 & 119.29 \\
\hline 2097.63 & 33.41 & 317.68 & 92.62 & 135.47 \\
\hline 2099.80 & 75.09 & 178.09 & 41.31 & 117.69 \\
\hline 2101.97 & 61.69 & 243.79 & 55.11 & 121.72 \\
\hline 2104.14 & 54.72 & 147.07 & 37.50 & 134.21 \\
\hline 2106.30 & 62.93 & 281.42 & 63.57 & 110.95 \\
\hline 2108.47 & 53.31 & 239.31 & 51.10 & 100.64 \\
\hline 2110.64 & 68.48 & 288.64 & 56.43 & 103.02 \\
\hline 2112.80 & 59.47 & 292.39 & 57.03 & 91.52 \\
\hline 2114.97 & 40.18 & 257.25 & 56.30 & 124.70 \\
\hline 2117.13 & 65.03 & 210.98 & 48.74 & 107.22 \\
\hline 2119.30 & 56.15 & 206.72 & 49.18 & 108.33 \\
\hline 2121.46 & 49.25 & 325.63 & 71.48 & 93.60 \\
\hline
\end{tabular}


Supplementary Information and Figures

\begin{tabular}{|c|c|c|c|c|}
\hline 2123.62 & 78.23 & 156.89 & 41.33 & 48.65 \\
\hline 2125.78 & 72.78 & 120.15 & 35.80 & 89.80 \\
\hline 2127.94 & 53.86 & 125.31 & 37.65 & 100.80 \\
\hline 2130.10 & 84.46 & 328.52 & 73.19 & 63.61 \\
\hline 2132.26 & 74.84 & 310.23 & 81.74 & 113.30 \\
\hline 2134.42 & 74.70 & 336.37 & 71.89 & 99.67 \\
\hline 2136.57 & 48.59 & 182.60 & 48.94 & 94.68 \\
\hline 2138.73 & 47.69 & 288.14 & 54.92 & 106.33 \\
\hline 2140.89 & 52.98 & 269.08 & 55.21 & 88.88 \\
\hline 2143.04 & 49.04 & 223.01 & 58.43 & 96.70 \\
\hline 2145.20 & 75.02 & 169.22 & 49.78 & 92.39 \\
\hline 2147.35 & 69.33 & 176.56 & 54.40 & 115.71 \\
\hline 2149.50 & 51.82 & 285.30 & 64.36 & 106.56 \\
\hline 2151.65 & 90.33 & 287.12 & 73.98 & 94.96 \\
\hline 2153.80 & 58.96 & 107.45 & 36.31 & 128.16 \\
\hline 2155.95 & 54.37 & 228.17 & 64.47 & 123.32 \\
\hline 2158.10 & 63.81 & 299.64 & 81.60 & 121.65 \\
\hline 2160.25 & 61.61 & 243.21 & 51.36 & 138.81 \\
\hline 2162.40 & 54.09 & 306.21 & 69.93 & 147.05 \\
\hline 2164.55 & 58.49 & 321.33 & 72.76 & 87.01 \\
\hline 2166.69 & 85.12 & 191.47 & 55.79 & 123.01 \\
\hline 2168.84 & 16.21 & 252.46 & 85.86 & 93.14 \\
\hline 2170.98 & 83.28 & 246.49 & 66.76 & 117.91 \\
\hline 2173.13 & 80.22 & 136.45 & 52.23 & 109.26 \\
\hline 2175.27 & 55.13 & 219.00 & 62.44 & 108.40 \\
\hline 2177.41 & 77.76 & 172.74 & 50.41 & 124.75 \\
\hline 2179.55 & 56.56 & 219.31 & 58.62 & 75.71 \\
\hline 2181.69 & 64.18 & 85.87 & 41.59 & 100.94 \\
\hline 2183.83 & 87.42 & 234.93 & 79.10 & 112.62 \\
\hline 2185.97 & 46.63 & 201.05 & 55.78 & 99.03 \\
\hline 2188.11 & 75.86 & 124.94 & 52.66 & 59.69 \\
\hline 2190.25 & 59.96 & 190.25 & 66.61 & 87.12 \\
\hline 2192.38 & 36.97 & 238.08 & 73.69 & 111.14 \\
\hline 2194.52 & 82.56 & 245.08 & 86.30 & 60.75 \\
\hline 2196.66 & 64.46 & 132.60 & 71.68 & 86.17 \\
\hline 2198.79 & 35.42 & 164.15 & 54.10 & 88.70 \\
\hline 2200.92 & 19.91 & 260.41 & 71.29 & 131.99 \\
\hline 2203.06 & 50.76 & 287.32 & 81.47 & 108.33 \\
\hline 2205.19 & 79.38 & 226.20 & 87.09 & 122.40 \\
\hline 2207.32 & 45.41 & 279.90 & 78.72 & 159.36 \\
\hline 2209.45 & 84.66 & 88.43 & 49.54 & 112.58 \\
\hline 2211.58 & 52.77 & 249.44 & 79.40 & 70.14 \\
\hline 2213.71 & 48.85 & 102.16 & 53.02 & 124.58 \\
\hline 2215.84 & 55.79 & 161.84 & 60.58 & 95.80 \\
\hline
\end{tabular}


Supplementary Information and Figures

\begin{tabular}{|c|c|c|c|c|}
\hline 2217.96 & 60.74 & 69.55 & 51.34 & 89.20 \\
\hline 2220.09 & 27.82 & 58.00 & 57.47 & 140.25 \\
\hline 2222.22 & 55.25 & 226.53 & 80.43 & 104.95 \\
\hline 2224.34 & 80.44 & 88.83 & 57.76 & 109.13 \\
\hline 2226.47 & 59.95 & 194.15 & 76.66 & 94.38 \\
\hline 2228.59 & 77.72 & 242.28 & 80.59 & 127.53 \\
\hline 2230.71 & 53.76 & 142.31 & 75.72 & 114.98 \\
\hline 2232.84 & 91.75 & 163.29 & 73.86 & 96.67 \\
\hline 2234.96 & 41.11 & 304.68 & 118.59 & 101.06 \\
\hline 2237.08 & 68.08 & 345.49 & 113.20 & 134.33 \\
\hline 2239.20 & 86.06 & 122.30 & 77.57 & 127.62 \\
\hline 2241.32 & 20.64 & 141.77 & 80.51 & 140.65 \\
\hline 2243.43 & 48.44 & 185.02 & 87.21 & 98.85 \\
\hline 2245.55 & 88.85 & 236.92 & 95.35 & 124.65 \\
\hline 2247.67 & 33.57 & 209.31 & 103.59 & 133.54 \\
\hline 2249.78 & 52.04 & 318.35 & 117.00 & 181.88 \\
\hline 2251.90 & 60.58 & 207.83 & 95.11 & 190.77 \\
\hline 2254.01 & 68.72 & 326.05 & 121.59 & 161.91 \\
\hline 2256.13 & 33.98 & 273.83 & 109.37 & 181.26 \\
\hline 2258.24 & 56.43 & 310.81 & 117.85 & 203.57 \\
\hline 2260.35 & 73.90 & 296.05 & 131.06 & 205.04 \\
\hline 2262.46 & 35.57 & 108.50 & 100.31 & 175.04 \\
\hline 2264.57 & 65.75 & 270.32 & 120.69 & 169.86 \\
\hline 2266.68 & 65.34 & 268.58 & 122.87 & 125.25 \\
\hline 2268.79 & 75.24 & 278.33 & 136.32 & 178.43 \\
\hline 2270.90 & 80.84 & 281.04 & 133.61 & 191.90 \\
\hline 2273.01 & 28.04 & 265.22 & 139.00 & 192.60 \\
\hline 2275.11 & 76.20 & 255.48 & 139.55 & 184.09 \\
\hline 2277.22 & 48.66 & 302.14 & 178.50 & 139.43 \\
\hline 2279.32 & 53.03 & 191.59 & 130.32 & 190.37 \\
\hline 2281.43 & 80.46 & 294.49 & 169.91 & 112.87 \\
\hline 2283.53 & 65.73 & 193.49 & 133.47 & 124.42 \\
\hline 2285.63 & 67.86 & 269.25 & 159.89 & 131.53 \\
\hline 2287.74 & 87.65 & 318.77 & 162.75 & 148.06 \\
\hline 2289.84 & 54.09 & 323.28 & 175.45 & 139.01 \\
\hline 2291.94 & 42.38 & 324.22 & 180.11 & 113.83 \\
\hline 2294.04 & 69.82 & 289.72 & 176.43 & 107.13 \\
\hline 2296.14 & 75.31 & 129.93 & 147.53 & 99.81 \\
\hline 2298.23 & 51.31 & 254.60 & 174.93 & 109.32 \\
\hline 2300.33 & 84.85 & 186.91 & 171.52 & 84.62 \\
\hline 2302.43 & 51.40 & 328.70 & 196.39 & 118.46 \\
\hline 2304.52 & 89.15 & 119.58 & 169.22 & 93.09 \\
\hline 2306.62 & 55.90 & 137.39 & 173.79 & 99.02 \\
\hline 2308.71 & 62.85 & 328.86 & 212.39 & 111.86 \\
\hline
\end{tabular}




\section{Supplementary Information and Figures}

\begin{tabular}{|c|c|c|c|c|}
\hline 2310.81 & 82.20 & 148.12 & 192.62 & 105.65 \\
\hline 2312.90 & 22.50 & 285.41 & 215.72 & 101.11 \\
\hline 2314.99 & 62.40 & 326.24 & 235.76 & 130.67 \\
\hline 2317.08 & 69.69 & 318.67 & 247.36 & 108.00 \\
\hline 2319.17 & 65.29 & 226.20 & 241.07 & 93.11 \\
\hline 2321.26 & 33.18 & 305.36 & 275.17 & 68.38 \\
\hline 2323.35 & 78.28 & 217.10 & 254.31 & 86.89 \\
\hline 2325.44 & 114.72 & 220.35 & 269.28 & 80.32 \\
\hline 2327.53 & 74.46 & 177.74 & 279.69 & 173.62 \\
\hline 2329.61 & 135.84 & 253.96 & 292.57 & 154.85 \\
\hline 2331.70 & 153.23 & 361.44 & 315.77 & 116.41 \\
\hline 2333.78 & 123.51 & 663.22 & 373.28 & 111.59 \\
\hline 2335.87 & 77.64 & 587.55 & 349.79 & 124.10 \\
\hline 2337.95 & 50.52 & 437.14 & 355.27 & 94.28 \\
\hline 2340.03 & 104.74 & 446.63 & 383.62 & 78.48 \\
\hline 2342.12 & 58.27 & 192.15 & 366.40 & 76.22 \\
\hline 2344.20 & 46.05 & 263.64 & 401.81 & 78.17 \\
\hline 2346.28 & 103.47 & 321.32 & 427.52 & 123.20 \\
\hline 2348.36 & 50.84 & 355.53 & 463.17 & 161.85 \\
\hline 2350.44 & 75.61 & 380.89 & 477.49 & 119.38 \\
\hline 2352.51 & 78.22 & 267.50 & 486.54 & 160.78 \\
\hline 2354.59 & 107.19 & 253.13 & 513.79 & 146.75 \\
\hline 2356.67 & 89.65 & 268.66 & 553.97 & 157.85 \\
\hline 2358.74 & 68.81 & 268.21 & 566.29 & 163.32 \\
\hline 2360.82 & 104.37 & 374.78 & 621.34 & 119.77 \\
\hline 2362.89 & 77.13 & 264.60 & 625.76 & 137.23 \\
\hline 2364.97 & 93.39 & 314.96 & 670.51 & 126.98 \\
\hline 2367.04 & 98.70 & 376.13 & 715.23 & 152.79 \\
\hline 2369.11 & 86.55 & 190.32 & 707.19 & 155.92 \\
\hline 2371.18 & 66.81 & 191.32 & 737.28 & 141.18 \\
\hline 2373.25 & 52.71 & 187.70 & 755.03 & 120.21 \\
\hline 2375.32 & 86.76 & 392.17 & 829.42 & 101.31 \\
\hline 2377.39 & 60.76 & 328.50 & 818.08 & 100.03 \\
\hline 2379.46 & 70.81 & 195.84 & 818.94 & 128.47 \\
\hline 2381.53 & 66.41 & 347.07 & 854.26 & 143.59 \\
\hline 2383.60 & 87.90 & 347.79 & 869.22 & 117.53 \\
\hline 2385.66 & 85.50 & 366.13 & 913.82 & 84.49 \\
\hline 2387.73 & 107.64 & 255.91 & 885.34 & 127.37 \\
\hline 2389.79 & 65.18 & 193.39 & 887.40 & 105.66 \\
\hline 2391.86 & 94.28 & 290.35 & 908.27 & 135.79 \\
\hline 2393.92 & 75.52 & 337.69 & 932.46 & 130.68 \\
\hline 2395.98 & 102.90 & 320.36 & 954.89 & 115.64 \\
\hline 2398.04 & 55.39 & 385.39 & 991.95 & 75.52 \\
\hline 2400.10 & 69.48 & 248.63 & 980.68 & 81.92 \\
\hline
\end{tabular}


Supplementary Information and Figures

\begin{tabular}{|c|c|c|c|c|}
\hline 2402.16 & 99.57 & 368.40 & 1035.58 & 63.74 \\
\hline 2404.22 & 92.45 & 245.06 & 1027.11 & 92.63 \\
\hline 2406.28 & 84.19 & 386.21 & 1067.38 & 82.49 \\
\hline 2408.34 & 63.67 & 375.76 & 1084.31 & 66.02 \\
\hline 2410.40 & 91.30 & 309.43 & 1084.25 & 42.87 \\
\hline 2412.45 & 60.38 & 240.00 & 1087.35 & 47.94 \\
\hline 2414.51 & 85.02 & 380.37 & 1136.35 & 86.73 \\
\hline 2416.56 & 66.59 & 196.71 & 1101.92 & 65.33 \\
\hline 2418.62 & 77.03 & 305.57 & 1115.79 & 102.66 \\
\hline 2420.67 & 70.70 & 370.89 & 1134.69 & 80.10 \\
\hline 2422.72 & 63.78 & 343.86 & 1171.49 & 100.66 \\
\hline 2424.77 & 43.36 & 230.84 & 1169.06 & 94.78 \\
\hline 2426.82 & 49.48 & 297.73 & 1239.43 & 76.98 \\
\hline 2428.87 & 61.41 & 297.55 & 1242.87 & 69.24 \\
\hline 2430.92 & 11.68 & 158.95 & 1277.61 & 72.63 \\
\hline 2432.97 & 70.16 & 216.18 & 1335.85 & 62.73 \\
\hline 2435.02 & 67.98 & 220.70 & 1411.42 & 53.75 \\
\hline 2437.07 & 16.46 & 306.18 & 1539.46 & 81.68 \\
\hline 2439.11 & 29.53 & 313.17 & 1609.94 & 92.63 \\
\hline 2441.16 & 78.15 & 128.70 & 1635.38 & 84.60 \\
\hline 2443.20 & 48.42 & 151.20 & 1721.95 & 118.38 \\
\hline 2445.25 & 62.40 & 308.63 & 1834.10 & 120.44 \\
\hline 2447.29 & 29.72 & 325.52 & 1922.87 & 103.35 \\
\hline 2449.33 & 48.94 & 145.88 & 1930.55 & 111.83 \\
\hline 2451.38 & 70.36 & 253.03 & 1987.26 & 132.29 \\
\hline 2453.42 & 64.48 & 215.43 & 2018.24 & 113.60 \\
\hline 2455.46 & 82.90 & 128.03 & 2013.25 & 84.39 \\
\hline 2457.50 & 85.07 & 317.16 & 2065.86 & 95.38 \\
\hline 2459.54 & 55.29 & 261.94 & 2043.34 & 136.15 \\
\hline 2461.57 & 64.91 & 279.46 & 2055.58 & 160.67 \\
\hline 2463.61 & 86.97 & 323.38 & 2044.16 & 144.76 \\
\hline 2465.65 & 85.39 & 291.71 & 2035.08 & 144.23 \\
\hline 2467.68 & 33.06 & 208.75 & 2004.32 & 134.49 \\
\hline 2469.72 & 75.38 & 271.31 & 2034.64 & 154.59 \\
\hline 2471.75 & 59.75 & 281.58 & 2044.95 & 150.93 \\
\hline 2473.79 & 71.77 & 317.34 & 2072.27 & 162.91 \\
\hline 2475.82 & 51.38 & 336.09 & 2109.68 & 145.64 \\
\hline 2477.85 & 83.30 & 277.59 & 2153.53 & 159.88 \\
\hline 2479.88 & 67.27 & 142.11 & 2197.01 & 152.94 \\
\hline 2481.91 & 81.59 & 269.86 & 2260.90 & 134.96 \\
\hline 2483.94 & 60.25 & 128.08 & 2282.91 & 149.65 \\
\hline 2485.97 & 55.82 & 212.93 & 2328.10 & 202.50 \\
\hline 2488.00 & 71.89 & 301.10 & 2381.31 & 147.17 \\
\hline 2490.03 & 70.76 & 308.29 & 2355.63 & 152.34 \\
\hline
\end{tabular}


Supplementary Information and Figures

\begin{tabular}{|c|c|c|c|c|}
\hline 2492.06 & 35.23 & 343.41 & 2390.02 & 161.39 \\
\hline 2494.08 & 115.84 & 294.10 & 2305.74 & 178.79 \\
\hline 2496.11 & 87.06 & 217.21 & 2276.75 & 164.51 \\
\hline 2498.13 & 31.53 & 164.10 & 2272.37 & 157.18 \\
\hline 2500.16 & 59.95 & 262.82 & 2308.16 & 178.23 \\
\hline 2502.18 & 89.22 & 272.64 & 2360.35 & 199.03 \\
\hline 2504.20 & 101.74 & 314.47 & 2407.37 & 187.94 \\
\hline 2506.22 & 14.50 & 288.46 & 2538.05 & 147.62 \\
\hline 2508.25 & 81.28 & 196.46 & 2560.21 & 175.17 \\
\hline 2510.27 & 62.05 & 315.80 & 2649.63 & 170.32 \\
\hline 2512.29 & 72.67 & 293.51 & 2683.49 & 163.04 \\
\hline 2514.30 & 87.09 & 173.74 & 2639.61 & 136.20 \\
\hline 2516.32 & 78.56 & 300.73 & 2611.80 & 172.29 \\
\hline 2518.34 & 74.58 & 240.98 & 2515.99 & 163.94 \\
\hline 2520.36 & 68.50 & 181.65 & 2411.91 & 143.81 \\
\hline 2522.37 & 54.02 & 264.24 & 2334.93 & 135.87 \\
\hline 2524.39 & 54.75 & 143.76 & 2236.92 & 70.71 \\
\hline 2526.40 & 52.17 & 123.24 & 2163.81 & 119.99 \\
\hline 2528.41 & 77.59 & 157.51 & 2116.10 & 129.94 \\
\hline 2530.43 & 70.12 & 195.24 & 2086.36 & 126.91 \\
\hline 2532.44 & 64.79 & 154.41 & 2051.39 & 149.28 \\
\hline 2534.45 & 66.27 & 227.82 & 2058.01 & 103.17 \\
\hline 2536.46 & 50.79 & 200.82 & 2056.60 & 120.96 \\
\hline 2538.47 & 26.32 & 192.23 & 2027.73 & 101.06 \\
\hline 2540.48 & 66.85 & 261.03 & 2034.85 & 130.18 \\
\hline 2542.49 & 59.73 & 117.95 & 1982.75 & 86.15 \\
\hline 2544.50 & 44.26 & 264.51 & 1944.54 & 123.49 \\
\hline 2546.50 & 18.43 & 173.73 & 1849.33 & 114.27 \\
\hline 2548.51 & 66.42 & 105.43 & 1744.93 & 82.62 \\
\hline 2550.51 & 71.14 & 243.07 & 1671.99 & 119.28 \\
\hline 2552.52 & 69.88 & 254.62 & 1575.95 & 86.10 \\
\hline 2554.52 & 42.11 & 211.48 & 1463.67 & 114.93 \\
\hline 2556.53 & 18.14 & 205.38 & 1385.80 & 108.42 \\
\hline 2558.53 & 38.78 & 278.34 & 1290.93 & 109.41 \\
\hline 2560.53 & 50.71 & 211.40 & 1204.76 & 128.91 \\
\hline 2562.53 & 88.00 & 229.84 & 1143.39 & 95.67 \\
\hline 2564.53 & 31.84 & 219.90 & 1094.78 & 108.49 \\
\hline 2566.53 & 71.87 & 185.71 & 1037.14 & 134.32 \\
\hline 2568.53 & 64.91 & 234.65 & 1005.21 & 122.60 \\
\hline 2570.53 & 77.95 & 269.86 & 978.20 & 178.35 \\
\hline 2572.52 & 84.09 & 239.51 & 950.60 & 157.79 \\
\hline 2574.52 & 53.18 & 192.10 & 910.36 & 138.10 \\
\hline 2576.52 & 54.93 & 136.47 & 890.02 & 178.47 \\
\hline 2578.51 & 77.17 & 262.37 & 891.05 & 142.39 \\
\hline
\end{tabular}


Supplementary Information and Figures

\begin{tabular}{|c|c|c|c|c|}
\hline 2580.51 & 30.67 & 278.37 & 876.75 & 175.27 \\
\hline 2582.50 & 62.61 & 208.70 & 861.51 & 158.16 \\
\hline 2584.49 & 83.66 & 149.46 & 848.24 & 132.10 \\
\hline 2586.48 & 57.90 & 242.30 & 861.24 & 125.39 \\
\hline 2588.48 & 80.30 & 181.66 & 861.30 & 151.86 \\
\hline 2590.47 & 45.80 & 230.10 & 886.13 & 155.87 \\
\hline 2592.46 & 90.50 & 308.31 & 936.03 & 170.84 \\
\hline 2594.45 & 73.95 & 319.12 & 941.40 & 176.06 \\
\hline 2596.43 & 69.20 & 192.23 & 951.83 & 183.68 \\
\hline 2598.42 & 87.10 & 114.30 & 967.29 & 180.72 \\
\hline 2600.41 & 93.55 & 291.85 & 1030.46 & 161.27 \\
\hline 2602.40 & 103.80 & 177.53 & 1045.92 & 167.62 \\
\hline 2604.38 & 83.30 & 325.72 & 1099.52 & 193.07 \\
\hline 2606.37 & 66.31 & 219.14 & 1076.59 & 173.53 \\
\hline 2608.35 & 55.16 & 194.60 & 1060.22 & 197.59 \\
\hline 2610.33 & 63.81 & 226.96 & 1033.12 & 191.71 \\
\hline 2612.32 & 72.22 & 268.89 & 1003.68 & 168.34 \\
\hline 2614.30 & 65.37 & 315.12 & 969.85 & 195.41 \\
\hline 2616.28 & 66.18 & 283.96 & 915.68 & 129.40 \\
\hline 2618.26 & 40.73 & 321.25 & 877.65 & 142.38 \\
\hline 2620.24 & 87.44 & 204.58 & 824.24 & 131.63 \\
\hline 2622.22 & 65.99 & 305.71 & 809.11 & 100.77 \\
\hline 2624.20 & 4.25 & 318.07 & 757.34 & 141.93 \\
\hline 2626.17 & 57.90 & 207.50 & 712.04 & 142.57 \\
\hline 2628.15 & 65.16 & 206.79 & 684.74 & 174.39 \\
\hline 2630.13 & 110.02 & 227.34 & 648.21 & 134.95 \\
\hline 2632.10 & 25.02 & 119.55 & 609.34 & 118.36 \\
\hline 2634.08 & 53.93 & 325.17 & 622.44 & 154.74 \\
\hline 2636.05 & 106.08 & 192.00 & 576.81 & 115.51 \\
\hline 2638.02 & 60.59 & 130.07 & 543.71 & 96.98 \\
\hline 2639.99 & 78.74 & 212.18 & 539.27 & 99.11 \\
\hline 2641.97 & 62.65 & 142.04 & 506.04 & 137.10 \\
\hline 2643.94 & 85.41 & 260.30 & 505.17 & 125.33 \\
\hline 2645.91 & 52.41 & 289.66 & 492.24 & 144.92 \\
\hline 2647.88 & 103.92 & 299.04 & 485.71 & 106.56 \\
\hline 2649.85 & 47.82 & 295.00 & 459.27 & 104.51 \\
\hline 2651.81 & 10.07 & 200.89 & 437.91 & 135.85 \\
\hline 2653.78 & 76.73 & 123.91 & 402.01 & 110.65 \\
\hline 2655.75 & 39.63 & 347.98 & 426.77 & 118.05 \\
\hline 2657.71 & 69.23 & 304.21 & 411.14 & 78.75 \\
\hline 2659.68 & 83.59 & 327.07 & 396.74 & 83.56 \\
\hline 2661.64 & 71.39 & 225.65 & 356.94 & 105.41 \\
\hline 2663.61 & 88.55 & 309.73 & 355.47 & 117.38 \\
\hline 2665.57 & 68.45 & 358.76 & 365.41 & 133.58 \\
\hline
\end{tabular}




\section{Supplementary Information and Figures}

\begin{tabular}{|c|c|c|c|c|}
\hline 2667.53 & 51.35 & 165.73 & 320.91 & 80.34 \\
\hline 2669.49 & 63.25 & 201.28 & 309.14 & 85.25 \\
\hline 2671.45 & 29.80 & 218.27 & 303.27 & 99.76 \\
\hline 2673.41 & 68.15 & 289.80 & 302.94 & 95.58 \\
\hline 2675.37 & 70.70 & 349.77 & 315.41 & 113.90 \\
\hline 2677.33 & 61.70 & 343.13 & 303.44 & 78.06 \\
\hline 2679.29 & 67.14 & 267.33 & 268.88 & 61.83 \\
\hline 2681.25 & 68.69 & 348.13 & 287.64 & 81.61 \\
\hline 2683.20 & 93.34 & 282.79 & 254.91 & 104.37 \\
\hline 2685.16 & 78.23 & 189.55 & 235.64 & 38.74 \\
\hline 2687.11 & 71.78 & 136.26 & 218.77 & 69.05 \\
\hline 2689.07 & 58.62 & 299.78 & 221.81 & 83.18 \\
\hline 2691.02 & 92.97 & 288.63 & 222.94 & 94.60 \\
\hline 2692.97 & 71.96 & 348.73 & 225.97 & 105.21 \\
\hline 2694.92 & 49.15 & 365.59 & 216.01 & 112.53 \\
\hline 2696.88 & 65.84 & 377.92 & 206.74 & 114.91 \\
\hline 2698.83 & 68.48 & 305.94 & 208.41 & 146.93 \\
\hline 2700.78 & 88.37 & 242.14 & 169.84 & 127.04 \\
\hline 2702.73 & 76.61 & 167.38 & 162.27 & 124.51 \\
\hline 2704.67 & 72.89 & 152.36 & 156.54 & 104.17 \\
\hline 2706.62 & 79.48 & 298.72 & 163.60 & 119.39 \\
\hline 2708.57 & 73.96 & 347.72 & 186.84 & 98.01 \\
\hline 2710.51 & 98.84 & 236.27 & 146.24 & 79.01 \\
\hline 2712.46 & 45.18 & 216.42 & 144.34 & 80.53 \\
\hline 2714.40 & 42.21 & 218.14 & 140.73 & 60.88 \\
\hline 2716.35 & 93.69 & 343.56 & 160.60 & 61.95 \\
\hline 2718.29 & 83.17 & 281.00 & 140.50 & 117.40 \\
\hline 2720.23 & 62.89 & 363.31 & 158.06 & 121.96 \\
\hline 2722.18 & 64.42 & 220.80 & 117.60 & 121.37 \\
\hline 2724.12 & 64.34 & 218.99 & 118.89 & 87.37 \\
\hline 2726.06 & 68.12 & 333.07 & 122.69 & 90.87 \\
\hline 2728.00 & 35.84 & 312.05 & 122.59 & 73.37 \\
\hline 2729.94 & 62.26 & 356.29 & 130.89 & 77.02 \\
\hline 2731.88 & 39.23 & 132.63 & 91.82 & 83.81 \\
\hline 2733.81 & 75.50 & 259.66 & 100.22 & 88.90 \\
\hline 2735.75 & 66.06 & 293.07 & 102.58 & 91.34 \\
\hline 2737.69 & 91.03 & 203.45 & 89.68 & 113.18 \\
\hline 2739.62 & 69.24 & 348.09 & 111.81 & 140.46 \\
\hline 2741.56 & 68.60 & 277.28 & 97.41 & 118.19 \\
\hline 2743.49 & 76.46 & 141.32 & 76.84 & 98.21 \\
\hline 2745.42 & 22.57 & 339.37 & 128.10 & 99.88 \\
\hline 2747.36 & 70.78 & 272.00 & 96.00 & 122.26 \\
\hline 2749.29 & 51.83 & 306.44 & 91.80 & 79.93 \\
\hline 2751.22 & 57.93 & 326.72 & 93.99 & 90.94 \\
\hline
\end{tabular}


Supplementary Information and Figures

\begin{tabular}{|c|c|c|c|c|}
\hline 2753.15 & 60.49 & 272.32 & 82.82 & 113.70 \\
\hline 2755.08 & 69.04 & 339.82 & 101.82 & 145.91 \\
\hline 2757.01 & 84.48 & 129.13 & 62.41 & 104.36 \\
\hline 2758.94 & 56.38 & 343.94 & 98.61 & 91.56 \\
\hline 2760.86 & 58.67 & 331.17 & 93.24 & 146.90 \\
\hline 2762.79 & 77.72 & 323.76 & 83.00 & 112.19 \\
\hline 2764.72 & 58.16 & 175.90 & 60.76 & 147.08 \\
\hline 2766.64 & 96.75 & 246.84 & 70.73 & 108.31 \\
\hline 2768.57 & 66.43 & 315.67 & 118.65 & 117.20 \\
\hline 2770.49 & 35.02 & 184.63 & 60.22 & 54.72 \\
\hline 2772.41 & 71.00 & 170.16 & 63.34 & 121.13 \\
\hline 2774.34 & 69.98 & 313.93 & 75.04 & 123.46 \\
\hline 2776.26 & 75.66 & 305.87 & 85.43 & 113.07 \\
\hline 2778.18 & 64.99 & 271.28 & 80.63 & 112.48 \\
\hline 2780.10 & 62.81 & 76.50 & 41.15 & 116.24 \\
\hline 2782.02 & 55.33 & 67.07 & 43.71 & 114.25 \\
\hline 2783.94 & 87.56 & 270.94 & 90.91 & 72.45 \\
\hline 2785.86 & 95.22 & 263.18 & 84.60 & 86.15 \\
\hline 2787.77 & 40.44 & 244.63 & 64.03 & 67.59 \\
\hline 2789.69 & 88.15 & 260.79 & 63.69 & 75.44 \\
\hline 2791.61 & 72.36 & 118.18 & 46.98 & 79.39 \\
\hline 2793.52 & 91.12 & 198.03 & 56.57 & 31.02 \\
\hline 2795.44 & 94.58 & 29.61 & 35.40 & 110.67 \\
\hline 2797.35 & 59.33 & 182.55 & 54.79 & 95.35 \\
\hline 2799.26 & 73.24 & 109.34 & 43.44 & 122.83 \\
\hline 2801.18 & 63.69 & 240.47 & 74.97 & 125.17 \\
\hline 2803.09 & 85.59 & 102.85 & 45.23 & 102.50 \\
\hline 2805.00 & 87.88 & 127.05 & 41.85 & 128.62 \\
\hline 2806.91 & 79.62 & 99.84 & 44.31 & 126.55 \\
\hline 2808.82 & 79.86 & 127.03 & 45.17 & 127.82 \\
\hline 2810.73 & 94.75 & 39.90 & 36.39 & 140.70 \\
\hline 2812.64 & 108.09 & 43.73 & 41.68 & 134.68 \\
\hline 2814.54 & 69.82 & 160.95 & 56.43 & 139.69 \\
\hline 2816.45 & 62.75 & 216.65 & 58.59 & 145.11 \\
\hline 2818.36 & 88.68 & 175.01 & 58.28 & 104.69 \\
\hline 2820.26 & 60.00 & 245.89 & 71.73 & 158.40 \\
\hline 2822.17 & 139.77 & 172.18 & 56.86 & 153.33 \\
\hline 2824.07 & 48.39 & 184.57 & 67.81 & 123.04 \\
\hline 2825.97 & 106.31 & 280.67 & 89.73 & 211.05 \\
\hline 2827.88 & 114.77 & 128.48 & 47.52 & 198.63 \\
\hline 2829.78 & 125.04 & 85.16 & 47.94 & 195.54 \\
\hline 2831.68 & 124.54 & 237.05 & 67.69 & 212.36 \\
\hline 2833.58 & 89.00 & 243.01 & 98.68 & 156.38 \\
\hline 2835.48 & 98.81 & 145.46 & 48.97 & 227.84 \\
\hline
\end{tabular}




\section{Supplementary Information and Figures}

\begin{tabular}{|c|c|c|c|c|}
\hline 2837.38 & 94.71 & 300.39 & 95.12 & 218.91 \\
\hline 2839.28 & 116.61 & 170.19 & 61.64 & 220.73 \\
\hline 2841.17 & 122.80 & 240.00 & 72.36 & 168.59 \\
\hline 2843.07 & 121.45 & 151.60 & 61.84 & 179.01 \\
\hline 2844.97 & 80.94 & 166.24 & 63.50 & 207.99 \\
\hline 2846.86 & 122.73 & 158.88 & 60.11 & 207.75 \\
\hline 2848.76 & 95.71 & 280.34 & 75.40 & 198.08 \\
\hline 2850.65 & 103.75 & 108.00 & 54.45 & 195.45 \\
\hline 2852.54 & 90.48 & 213.87 & 67.60 & 201.21 \\
\hline 2854.44 & 110.61 & 289.15 & 77.39 & 187.50 \\
\hline 2856.33 & 85.54 & 230.92 & 103.94 & 209.92 \\
\hline 2858.22 & 116.06 & 233.81 & 80.42 & 210.85 \\
\hline 2860.11 & 142.63 & 276.67 & 93.73 & 189.27 \\
\hline 2862.00 & 116.40 & 260.31 & 88.25 & 196.10 \\
\hline 2863.89 & 109.02 & 198.55 & 74.00 & 192.39 \\
\hline 2865.78 & 87.68 & 275.53 & 92.75 & 170.87 \\
\hline 2867.67 & 110.95 & 265.56 & 95.47 & 146.71 \\
\hline 2869.55 & 120.36 & 273.53 & 89.48 & 153.84 \\
\hline 2871.44 & 94.76 & 77.63 & 65.43 & 164.38 \\
\hline 2873.33 & 106.92 & 160.57 & 74.01 & 179.33 \\
\hline 2875.21 & 138.77 & 255.63 & 95.99 & 163.37 \\
\hline 2877.09 & 125.82 & 269.72 & 99.34 & 152.96 \\
\hline 2878.98 & 83.77 & 193.88 & 85.29 & 149.27 \\
\hline 2880.86 & 115.27 & 234.67 & 99.23 & 143.12 \\
\hline 2882.74 & 102.76 & 304.39 & 108.91 & 183.47 \\
\hline 2884.62 & 99.31 & 150.48 & 75.76 & 155.88 \\
\hline 2886.50 & 114.35 & 264.59 & 127.67 & 173.68 \\
\hline 2888.38 & 84.33 & 196.29 & 87.39 & 199.75 \\
\hline 2890.26 & 93.27 & 204.64 & 101.66 & 148.57 \\
\hline 2892.14 & 95.30 & 299.90 & 120.48 & 206.09 \\
\hline 2894.02 & 101.28 & 219.67 & 104.32 & 162.76 \\
\hline 2895.90 & 100.41 & 225.18 & 98.90 & 141.29 \\
\hline 2897.77 & 113.09 & 211.55 & 100.95 & 177.87 \\
\hline 2899.65 & 142.81 & 156.98 & 93.99 & 193.00 \\
\hline 2901.53 & 112.99 & 288.97 & 117.70 & 207.55 \\
\hline 2903.40 & 102.71 & 170.65 & 108.38 & 214.83 \\
\hline 2905.27 & 129.23 & 167.73 & 101.62 & 176.08 \\
\hline 2907.15 & 92.74 & 174.32 & 99.83 & 140.18 \\
\hline 2909.02 & 124.21 & 263.07 & 118.18 & 147.78 \\
\hline 2910.89 & 93.77 & 234.56 & 110.22 & 124.04 \\
\hline 2912.76 & 140.98 & 120.55 & 96.40 & 133.40 \\
\hline 2914.63 & 102.04 & 105.32 & 100.41 & 116.17 \\
\hline 2916.50 & 99.59 & 315.46 & 117.81 & 150.99 \\
\hline 2918.37 & 123.50 & 179.08 & 106.66 & 149.07 \\
\hline
\end{tabular}




\section{Supplementary Information and Figures}

\begin{tabular}{|c|c|c|c|c|}
\hline 2920.24 & 116.95 & 224.35 & 114.83 & 120.00 \\
\hline 2922.10 & 108.45 & 178.49 & 109.61 & 159.23 \\
\hline 2923.97 & 90.65 & 234.98 & 130.32 & 125.57 \\
\hline 2925.84 & 113.54 & 316.07 & 156.92 & 116.82 \\
\hline 2927.70 & 129.79 & 193.89 & 116.13 & 119.41 \\
\hline 2929.57 & 139.93 & 276.30 & 129.94 & 105.42 \\
\hline 2931.43 & 156.37 & 275.11 & 135.28 & 96.02 \\
\hline 2933.29 & 120.41 & 310.98 & 135.79 & 91.28 \\
\hline 2935.16 & 89.44 & 255.41 & 147.76 & 98.35 \\
\hline 2937.02 & 109.43 & 156.04 & 119.60 & 82.17 \\
\hline 2938.88 & 117.06 & 216.31 & 125.41 & 103.03 \\
\hline 2940.74 & 115.04 & 279.92 & 140.61 & 80.41 \\
\hline 2942.60 & 123.62 & 108.24 & 119.08 & 94.93 \\
\hline 2944.46 & 156.30 & 187.22 & 129.42 & 78.36 \\
\hline 2946.32 & 172.42 & 253.46 & 136.46 & 93.99 \\
\hline 2948.17 & 135.35 & 297.44 & 145.53 & 71.61 \\
\hline 2950.03 & 183.57 & 248.89 & 144.24 & 110.30 \\
\hline 2951.89 & 112.59 & 195.47 & 126.01 & 150.09 \\
\hline 2953.74 & 157.01 & 254.18 & 131.68 & 134.87 \\
\hline 2955.60 & 133.12 & 334.02 & 155.52 & 121.16 \\
\hline 2957.45 & 165.19 & 247.87 & 136.69 & 109.90 \\
\hline 2959.31 & 154.80 & 232.49 & 130.46 & 125.39 \\
\hline 2961.16 & 155.76 & 181.02 & 131.36 & 130.28 \\
\hline 2963.01 & 167.47 & 303.57 & 145.10 & 104.32 \\
\hline 2964.86 & 162.53 & 333.53 & 156.04 & 119.61 \\
\hline 2966.71 & 173.28 & 292.05 & 134.11 & 143.95 \\
\hline 2968.56 & 162.94 & 237.61 & 125.28 & 109.64 \\
\hline 2970.41 & 169.59 & 313.94 & 137.91 & 133.78 \\
\hline 2972.26 & 151.79 & 329.77 & 139.78 & 95.67 \\
\hline 2974.11 & 208.89 & 216.07 & 127.12 & 100.71 \\
\hline 2975.96 & 204.09 & 365.71 & 150.05 & 87.70 \\
\hline 2977.80 & 167.43 & 315.88 & 139.05 & 114.73 \\
\hline 2979.65 & 178.17 & 218.55 & 121.92 & 67.47 \\
\hline 2981.49 & 204.92 & 307.42 & 136.89 & 102.59 \\
\hline 2983.34 & 194.96 & 319.35 & 151.82 & 68.68 \\
\hline 2985.18 & 129.90 & 236.07 & 126.02 & 35.16 \\
\hline 2987.02 & 201.68 & 255.06 & 137.46 & 70.53 \\
\hline 2988.87 & 208.27 & 280.70 & 136.62 & 53.67 \\
\hline 2990.71 & 192.55 & 358.08 & 164.59 & 46.44 \\
\hline 2992.55 & 158.09 & 325.71 & 155.06 & 49.10 \\
\hline 2994.39 & 221.32 & 263.45 & 141.62 & 46.57 \\
\hline 2996.23 & 182.00 & 287.85 & 142.89 & 70.84 \\
\hline 2998.07 & 197.43 & 262.84 & 142.59 & 67.25 \\
\hline 2999.91 & 214.35 & 140.33 & 130.15 & 49.81 \\
\hline
\end{tabular}




\section{Supplementary Information and Figures}

\begin{tabular}{|c|c|c|c|c|}
\hline 3001.74 & 217.03 & 274.54 & 148.28 & 52.00 \\
\hline 3003.58 & 225.25 & 284.39 & 158.02 & 73.45 \\
\hline 3005.42 & 202.67 & 287.76 & 164.28 & 54.96 \\
\hline 3007.25 & 230.34 & 149.78 & 141.68 & 98.69 \\
\hline 3009.09 & 254.81 & 135.46 & 139.21 & 58.63 \\
\hline 3010.92 & 233.53 & 336.91 & 171.07 & 61.21 \\
\hline 3012.75 & 222.30 & 208.19 & 158.24 & 78.13 \\
\hline 3014.59 & 267.11 & 272.17 & 173.84 & 93.62 \\
\hline 3016.42 & 295.77 & 289.74 & 167.80 & 112.53 \\
\hline 3018.25 & 262.39 & 213.80 & 164.60 & 111.54 \\
\hline 3020.08 & 322.15 & 284.53 & 168.73 & 112.40 \\
\hline 3021.91 & 282.46 & 221.51 & 166.42 & 137.50 \\
\hline 3023.74 & 285.36 & 323.18 & 187.12 & 117.04 \\
\hline 3025.57 & 266.07 & 293.26 & 183.28 & 114.03 \\
\hline 3027.40 & 298.08 & 355.17 & 204.44 & 143.67 \\
\hline 3029.22 & 293.48 & 291.18 & 196.94 & 173.94 \\
\hline 3031.05 & 300.73 & 391.14 & 219.80 & 175.21 \\
\hline 3032.88 & 319.28 & 167.36 & 178.56 & 141.38 \\
\hline 3034.70 & 325.68 & 229.68 & 194.79 & 135.78 \\
\hline 3036.53 & 308.28 & 354.34 & 220.05 & 155.63 \\
\hline 3038.35 & 357.53 & 291.54 & 216.28 & 109.77 \\
\hline 3040.17 & 327.27 & 288.40 & 214.21 & 129.20 \\
\hline 3041.99 & 351.97 & 290.18 & 216.93 & 122.07 \\
\hline 3043.82 & 383.91 & 311.86 & 238.99 & 115.30 \\
\hline 3045.64 & 382.30 & 365.17 & 238.55 & 118.66 \\
\hline 3047.46 & 403.49 & 273.38 & 234.55 & 106.31 \\
\hline 3049.28 & 369.08 & 370.43 & 254.91 & 109.06 \\
\hline 3051.10 & 382.97 & 348.00 & 256.00 & 97.89 \\
\hline 3052.91 & 416.31 & 358.07 & 269.36 & 115.71 \\
\hline 3054.73 & 416.34 & 137.08 & 236.98 & 109.23 \\
\hline 3056.55 & 415.58 & 158.47 & 234.64 & 116.19 \\
\hline 3058.37 & 427.06 & 333.70 & 302.20 & 116.34 \\
\hline 3060.18 & 421.99 & 305.66 & 273.66 & 110.03 \\
\hline 3062.00 & 447.33 & 364.62 & 290.32 & 148.26 \\
\hline 3063.81 & 432.05 & 282.50 & 283.94 & 121.58 \\
\hline 3065.62 & 462.13 & 188.46 & 274.60 & 158.33 \\
\hline 3067.44 & 468.01 & 204.23 & 285.06 & 156.54 \\
\hline 3069.25 & 484.99 & 234.11 & 286.25 & 113.13 \\
\hline 3071.06 & 500.66 & 222.83 & 290.24 & 106.40 \\
\hline 3072.87 & 475.04 & 211.91 & 303.17 & 132.47 \\
\hline 3074.68 & 498.11 & 330.61 & 345.12 & 108.98 \\
\hline 3076.49 & 492.23 & 233.81 & 320.28 & 102.33 \\
\hline 3078.30 & 508.60 & 352.31 & 347.80 & 121.61 \\
\hline 3080.11 & 557.02 & 173.72 & 328.16 & 122.34 \\
\hline
\end{tabular}




\section{Supplementary Information and Figures}

\begin{tabular}{|c|c|c|c|c|}
\hline 3081.91 & 551.44 & 383.97 & 370.88 & 131.04 \\
\hline 3083.72 & 541.76 & 267.08 & 346.20 & 119.35 \\
\hline 3085.53 & 562.38 & 411.79 & 374.63 & 127.24 \\
\hline 3087.33 & 600.14 & 329.13 & 375.25 & 89.31 \\
\hline 3089.14 & 616.66 & 269.13 & 370.87 & 89.03 \\
\hline 3090.94 & 619.52 & 212.76 & 379.69 & 106.58 \\
\hline 3092.74 & 608.78 & 422.04 & 412.95 & 131.52 \\
\hline 3094.55 & 639.54 & 397.92 & 420.20 & 83.75 \\
\hline 3096.35 & 623.60 & 434.51 & 433.02 & 100.71 \\
\hline 3098.15 & 654.76 & 373.20 & 443.21 & 135.56 \\
\hline 3099.95 & 680.72 & 292.90 & 434.03 & 157.56 \\
\hline 3101.75 & 646.93 & 430.30 & 467.12 & 138.24 \\
\hline 3103.55 & 707.44 & 400.59 & 489.51 & 146.81 \\
\hline 3105.35 & 732.04 & 277.69 & 463.96 & 104.07 \\
\hline 3107.15 & 728.10 & 326.41 & 481.11 & 101.17 \\
\hline 3108.94 & 755.10 & 370.04 & 513.47 & 113.11 \\
\hline 3110.74 & 725.86 & 370.31 & 515.79 & 44.10 \\
\hline 3112.53 & 778.71 & 331.25 & 522.54 & 68.17 \\
\hline 3114.33 & 785.06 & 333.26 & 544.86 & 37.39 \\
\hline 3116.12 & 821.01 & 369.91 & 558.71 & 45.60 \\
\hline 3117.92 & 818.36 & 372.76 & 564.30 & 56.66 \\
\hline 3119.71 & 819.26 & 368.75 & 596.65 & 74.96 \\
\hline 3121.50 & 883.91 & 370.12 & 607.13 & 72.50 \\
\hline 3123.29 & 881.75 & 253.59 & 591.42 & 58.44 \\
\hline 3125.09 & 913.10 & 183.85 & 593.04 & 66.69 \\
\hline 3126.88 & 928.24 & 424.34 & 652.35 & 89.87 \\
\hline 3128.67 & 960.79 & 234.05 & 628.64 & 102.75 \\
\hline 3130.46 & 980.13 & 242.14 & 641.39 & 65.33 \\
\hline 3132.24 & 981.23 & 364.45 & 678.61 & 52.05 \\
\hline 3134.03 & 1014.17 & 283.36 & 681.26 & 49.07 \\
\hline 3135.82 & 1032.76 & 457.29 & 719.14 & 55.30 \\
\hline 3137.60 & 1036.60 & 367.29 & 717.36 & 70.86 \\
\hline 3139.39 & 1080.59 & 428.36 & 732.87 & 40.19 \\
\hline 3141.17 & 1096.13 & 516.15 & 772.62 & 7.06 \\
\hline 3142.96 & 1105.17 & 505.25 & 779.14 & 32.19 \\
\hline 3144.74 & 1189.85 & 480.99 & 786.29 & 27.86 \\
\hline 3146.53 & 1180.29 & 621.32 & 847.31 & 55.19 \\
\hline 3148.31 & 1217.88 & 628.17 & 839.49 & 86.12 \\
\hline 3150.09 & 1252.01 & 604.11 & 837.80 & 85.15 \\
\hline 3151.87 & 1283.15 & 562.18 & 847.39 & 96.73 \\
\hline 3153.65 & 1322.78 & 844.15 & 892.63 & 96.97 \\
\hline 3155.43 & 1386.27 & 932.65 & 916.95 & 104.41 \\
\hline 3157.21 & 1395.85 & 955.89 & 942.50 & 77.31 \\
\hline 3158.99 & 1430.53 & 1050.86 & 942.41 & 70.72 \\
\hline
\end{tabular}




\section{Supplementary Information and Figures}

\begin{tabular}{|c|c|c|c|c|}
\hline 3160.76 & 1456.11 & 1126.75 & 958.66 & 48.27 \\
\hline 3162.54 & 1493.64 & 1163.63 & 966.44 & 82.84 \\
\hline 3164.32 & 1547.22 & 1312.28 & 976.55 & 88.96 \\
\hline 3166.09 & 1560.10 & 1463.13 & 1001.30 & 87.68 \\
\hline 3167.87 & 1608.98 & 1572.30 & 1018.78 & 81.08 \\
\hline 3169.64 & 1642.46 & 1698.49 & 1022.06 & 63.67 \\
\hline 3171.42 & 1686.19 & 1943.81 & 1070.68 & 68.03 \\
\hline 3173.19 & 1775.01 & 2104.03 & 1067.32 & 102.79 \\
\hline 3174.96 & 1778.74 & 2121.51 & 1081.77 & 85.56 \\
\hline 3176.73 & 1798.97 & 2140.49 & 1092.05 & 59.64 \\
\hline 3178.50 & 1860.69 & 2329.84 & 1120.23 & 60.58 \\
\hline 3180.27 & 1898.62 & 2300.32 & 1135.64 & 69.08 \\
\hline 3182.04 & 1953.19 & 2127.42 & 1121.32 & 90.55 \\
\hline 3183.81 & 1984.86 & 2249.98 & 1155.03 & 62.78 \\
\hline 3185.58 & 2010.44 & 2272.50 & 1187.51 & 19.82 \\
\hline 3187.35 & 2113.21 & 2121.89 & 1176.29 & 31.27 \\
\hline 3189.11 & 2171.48 & 2126.78 & 1203.37 & 30.53 \\
\hline 3190.88 & 2185.65 & 2043.03 & 1211.18 & 34.66 \\
\hline 3192.65 & 2236.78 & 2122.46 & 1233.86 & 29.11 \\
\hline 3194.41 & 2281.10 & 2104.00 & 1261.00 & 38.17 \\
\hline 3196.17 & 2334.72 & 2021.51 & 1249.21 & 51.39 \\
\hline 3197.94 & 2369.34 & 2111.23 & 1279.99 & 56.08 \\
\hline 3199.70 & 2430.56 & 2291.45 & 1323.70 & 54.03 \\
\hline 3201.46 & 2456.72 & 2303.77 & 1323.58 & 50.70 \\
\hline 3203.22 & 2546.04 & 2397.02 & 1346.49 & 96.84 \\
\hline 3204.99 & 2557.01 & 2581.59 & 1365.33 & 57.36 \\
\hline 3206.75 & 2636.58 & 2893.99 & 1405.81 & 44.73 \\
\hline 3208.50 & 2668.50 & 2969.06 & 1418.02 & 66.11 \\
\hline 3210.26 & 2681.91 & 3252.49 & 1448.96 & 36.23 \\
\hline 3212.02 & 2802.28 & 3640.82 & 1510.77 & 92.96 \\
\hline 3213.78 & 2819.84 & 3822.36 & 1503.68 & 94.87 \\
\hline 3215.54 & 2880.81 & 4289.92 & 1562.55 & 112.99 \\
\hline 3217.29 & 2978.08 & 4614.34 & 1593.96 & 94.17 \\
\hline 3219.05 & 3029.44 & 4922.81 & 1616.47 & 99.04 \\
\hline 3220.80 & 3090.55 & 5163.23 & 1643.51 & 95.17 \\
\hline 3222.56 & 3177.52 & 5399.84 & 1679.42 & 110.79 \\
\hline 3224.31 & 3228.63 & 5498.53 & 1712.20 & 91.26 \\
\hline 3226.06 & 3279.05 & 5656.47 & 1760.17 & 104.26 \\
\hline 3227.81 & 3353.51 & 5591.06 & 1789.54 & 88.63 \\
\hline 3229.57 & 3426.17 & 5585.26 & 1845.19 & 112.79 \\
\hline 3231.32 & 3481.08 & 5491.92 & 1910.09 & 113.41 \\
\hline 3233.07 & 3515.10 & 5263.53 & 1943.03 & 146.91 \\
\hline 3234.82 & 3631.56 & 4931.82 & 1975.11 & 131.23 \\
\hline 3236.57 & 3712.17 & 4767.53 & 2025.68 & 149.13 \\
\hline
\end{tabular}




\section{Supplementary Information and Figures}

\begin{tabular}{|c|c|c|c|c|}
\hline 3238.31 & 3744.88 & 4567.79 & 2076.19 & 146.99 \\
\hline 3240.06 & 3887.49 & 4402.81 & 2134.46 & 106.65 \\
\hline 3241.81 & 3919.10 & 4168.93 & 2155.13 & 120.28 \\
\hline 3243.55 & 3965.81 & 4029.91 & 2188.84 & 127.08 \\
\hline 3245.30 & 4080.02 & 3887.70 & 2220.55 & 143.07 \\
\hline 3247.04 & 4137.93 & 4006.87 & 2298.89 & 126.27 \\
\hline 3248.79 & 4224.09 & 3910.77 & 2349.19 & 118.02 \\
\hline 3250.53 & 4320.35 & 3978.09 & 2374.96 & 149.33 \\
\hline 3252.27 & 4441.41 & 3762.91 & 2351.90 & 113.62 \\
\hline 3254.02 & 4523.17 & 3835.93 & 2390.88 & 128.41 \\
\hline 3255.76 & 4620.78 & 3874.10 & 2424.15 & 124.07 \\
\hline 3257.50 & 4709.49 & 4010.13 & 2444.45 & 168.15 \\
\hline 3259.24 & 4788.35 & 4076.41 & 2467.46 & 143.02 \\
\hline 3260.98 & 4827.91 & 4281.06 & 2492.69 & 146.53 \\
\hline 3262.72 & 4971.06 & 4578.09 & 2497.07 & 116.61 \\
\hline 3264.46 & 5054.87 & 4810.46 & 2516.87 & 118.03 \\
\hline 3266.19 & 5122.78 & 5384.13 & 2545.57 & 116.51 \\
\hline 3267.93 & 5215.09 & 5715.45 & 2517.88 & 110.45 \\
\hline 3269.67 & 5244.20 & 6248.06 & 2531.88 & 104.67 \\
\hline 3271.40 & 5347.50 & 6714.82 & 2526.05 & 98.61 \\
\hline 3273.14 & 5325.61 & 7274.10 & 2539.59 & 106.90 \\
\hline 3274.87 & 5412.32 & 8008.57 & 2586.09 & 87.37 \\
\hline 3276.60 & 5428.32 & 8467.42 & 2572.40 & 88.51 \\
\hline 3278.34 & 5426.83 & 8720.66 & 2553.73 & 91.52 \\
\hline 3280.07 & 5472.49 & 9013.94 & 2549.20 & 99.24 \\
\hline 3281.80 & 5467.99 & 9353.79 & 2567.91 & 101.70 \\
\hline 3283.53 & 5506.80 & 9443.53 & 2577.44 & 105.34 \\
\hline 3285.26 & 5412.31 & 9395.46 & 2582.94 & 100.58 \\
\hline 3286.99 & 5434.32 & 9223.63 & 2588.98 & 107.15 \\
\hline 3288.72 & 5369.97 & 9006.30 & 2606.72 & 78.72 \\
\hline 3290.45 & 5371.38 & 8511.03 & 2615.08 & 100.17 \\
\hline 3292.18 & 5350.99 & 7884.09 & 2607.62 & 95.57 \\
\hline 3293.90 & 5330.49 & 7397.66 & 2623.29 & 67.73 \\
\hline 3295.63 & 5319.75 & 6665.35 & 2611.96 & 45.01 \\
\hline 3297.35 & 5263.40 & 6252.67 & 2618.06 & 73.41 \\
\hline 3299.08 & 5274.86 & 5856.68 & 2647.92 & 95.22 \\
\hline 3300.80 & 5234.07 & 5559.83 & 2679.49 & 120.70 \\
\hline 3302.53 & 5250.62 & 5085.66 & 2636.16 & 85.99 \\
\hline 3304.25 & 5201.63 & 4870.44 & 2654.53 & 50.91 \\
\hline 3305.97 & 5169.39 & 4850.48 & 2695.83 & 81.37 \\
\hline 3307.69 & 5189.29 & 4536.69 & 2679.43 & 54.38 \\
\hline 3309.42 & 5155.05 & 4552.67 & 2723.83 & 120.00 \\
\hline 3311.14 & 5106.36 & 4487.95 & 2720.10 & 84.49 \\
\hline 3312.86 & 5133.96 & 4408.29 & 2730.93 & 96.58 \\
\hline
\end{tabular}




\section{Supplementary Information and Figures}

\begin{tabular}{|c|c|c|c|c|}
\hline 3314.57 & 5122.32 & 4664.69 & 2789.06 & 51.24 \\
\hline 3316.29 & 5138.53 & 4642.74 & 2789.60 & 63.62 \\
\hline 3318.01 & 5128.98 & 4703.60 & 2806.86 & 61.29 \\
\hline 3319.73 & 5122.59 & 4847.88 & 2858.06 & 47.35 \\
\hline 3321.44 & 5175.85 & 4825.75 & 2847.66 & 62.99 \\
\hline 3323.16 & 5137.65 & 4867.42 & 2860.89 & 101.29 \\
\hline 3324.87 & 5212.11 & 5021.43 & 2899.76 & 104.25 \\
\hline 3326.59 & 5202.47 & 5180.06 & 2917.79 & 82.54 \\
\hline 3328.30 & 5203.18 & 5276.26 & 2942.82 & 59.09 \\
\hline 3330.02 & 5255.68 & 5353.00 & 2977.96 & 106.51 \\
\hline 3331.73 & 5231.59 & 5369.95 & 2974.65 & 62.94 \\
\hline 3333.44 & 5303.55 & 5538.89 & 3023.89 & 81.06 \\
\hline 3335.15 & 5327.11 & 5491.08 & 3041.65 & 93.28 \\
\hline 3336.86 & 5321.32 & 5561.84 & 3076.11 & 74.83 \\
\hline 3338.57 & 5390.77 & 5663.22 & 3109.91 & 94.07 \\
\hline 3340.28 & 5394.13 & 5701.26 & 3115.51 & 22.01 \\
\hline 3341.99 & 5395.99 & 5686.83 & 3150.74 & 30.38 \\
\hline 3343.70 & 5426.85 & 5701.86 & 3171.37 & 71.49 \\
\hline 3345.41 & 5507.81 & 5693.90 & 3166.50 & 57.59 \\
\hline 3347.11 & 5534.02 & 5603.73 & 3154.13 & 73.31 \\
\hline 3348.82 & 5526.63 & 5868.56 & 3197.43 & 79.23 \\
\hline 3350.52 & 5636.59 & 5870.66 & 3208.36 & 71.87 \\
\hline 3352.23 & 5653.15 & 6008.92 & 3238.46 & 79.27 \\
\hline 3353.93 & 5667.11 & 6008.41 & 3225.35 & 63.63 \\
\hline 3355.63 & 5754.47 & 6105.40 & 3234.08 & 78.43 \\
\hline 3357.34 & 5772.53 & 6118.47 & 3243.61 & 85.39 \\
\hline 3359.04 & 5838.44 & 6124.21 & 3248.71 & 82.46 \\
\hline 3360.74 & 5845.90 & 6260.12 & 3280.90 & 99.72 \\
\hline 3362.44 & 5940.36 & 6409.76 & 3318.73 & 55.81 \\
\hline 3364.14 & 5977.42 & 6526.23 & 3341.93 & 82.48 \\
\hline 3365.84 & 6018.38 & 6477.15 & 3342.99 & 82.25 \\
\hline 3367.54 & 6050.00 & 6573.32 & 3377.92 & 82.64 \\
\hline 3369.24 & 6125.96 & 6768.79 & 3437.68 & 113.17 \\
\hline 3370.94 & 6182.92 & 6724.64 & 3428.08 & 84.24 \\
\hline 3372.63 & 6221.39 & 7059.99 & 3493.31 & 101.30 \\
\hline 3374.33 & 6273.20 & 7185.34 & 3534.00 & 49.39 \\
\hline 3376.02 & 6316.61 & 7322.12 & 3559.79 & 76.97 \\
\hline 3377.72 & 6427.48 & 7703.95 & 3626.89 & 115.47 \\
\hline 3379.41 & 6510.69 & 7842.61 & 3648.12 & 97.87 \\
\hline 3381.11 & 6469.06 & 8251.75 & 3730.98 & 54.38 \\
\hline 3382.80 & 6650.57 & 8454.63 & 3730.07 & 106.09 \\
\hline 3384.49 & 6638.83 & 8814.37 & 3787.37 & 57.49 \\
\hline 3386.18 & 6699.85 & 9093.20 & 3848.26 & 71.71 \\
\hline 3387.87 & 6772.32 & 9167.81 & 3875.65 & 90.12 \\
\hline
\end{tabular}




\section{Supplementary Information and Figures}

\begin{tabular}{|c|c|c|c|c|}
\hline 3389.56 & 6809.78 & 9382.76 & 3946.01 & 74.71 \\
\hline 3391.25 & 6881.35 & 9352.28 & 3971.31 & 117.08 \\
\hline 3392.94 & 6902.07 & 9092.90 & 3950.63 & 142.92 \\
\hline 3394.63 & 6976.23 & 9156.38 & 4001.23 & 128.40 \\
\hline 3396.32 & 7017.60 & 9104.19 & 4042.02 & 138.86 \\
\hline 3398.01 & 7038.42 & 8767.29 & 4040.55 & 143.15 \\
\hline 3399.69 & 7037.59 & 8713.82 & 4066.24 & 121.33 \\
\hline 3401.38 & 7050.26 & 8529.28 & 4063.50 & 121.33 \\
\hline 3403.06 & 7058.33 & 8511.00 & 4094.12 & 91.07 \\
\hline 3404.75 & 7020.00 & 8338.04 & 4096.82 & 99.57 \\
\hline 3406.43 & 7034.42 & 8217.68 & 4110.44 & 94.06 \\
\hline 3408.12 & 7031.84 & 8094.16 & 4106.77 & 100.27 \\
\hline 3409.80 & 6953.26 & 8114.34 & 4128.06 & 124.22 \\
\hline 3411.48 & 6995.08 & 7877.78 & 4102.72 & 125.85 \\
\hline 3413.16 & 6954.75 & 8013.39 & 4120.44 & 108.80 \\
\hline 3414.84 & 6891.68 & 8076.54 & 4119.34 & 104.78 \\
\hline 3416.52 & 6858.70 & 8225.35 & 4133.79 & 98.44 \\
\hline 3418.20 & 6828.52 & 8415.29 & 4115.05 & 65.37 \\
\hline 3419.88 & 6728.35 & 8464.09 & 4098.11 & 68.38 \\
\hline 3421.56 & 6729.52 & 8681.74 & 4117.00 & 105.36 \\
\hline 3423.24 & 6641.30 & 8620.53 & 4070.56 & 98.37 \\
\hline 3424.91 & 6610.07 & 8816.54 & 4076.32 & 47.27 \\
\hline 3426.59 & 6535.55 & 8788.56 & 4050.41 & 86.53 \\
\hline 3428.26 & 6471.62 & 8696.69 & 3989.96 & 77.27 \\
\hline 3429.94 & 6427.60 & 8535.66 & 3959.29 & 78.48 \\
\hline 3431.61 & 6335.38 & 8508.14 & 3931.28 & 74.37 \\
\hline 3433.29 & 6293.56 & 8215.62 & 3859.90 & 97.72 \\
\hline 3434.96 & 6227.14 & 7980.41 & 3828.96 & 129.96 \\
\hline 3436.63 & 6155.91 & 7567.29 & 3747.38 & 132.82 \\
\hline 3438.30 & 6085.59 & 7385.75 & 3687.97 & 172.21 \\
\hline 3439.97 & 6027.52 & 7148.70 & 3647.06 & 154.66 \\
\hline 3441.64 & 5893.31 & 6911.35 & 3591.82 & 134.44 \\
\hline 3443.31 & 5844.14 & 6619.58 & 3515.31 & 158.24 \\
\hline 3444.98 & 5762.77 & 6345.46 & 3442.10 & 165.56 \\
\hline 3446.65 & 5691.90 & 5919.60 & 3371.62 & 170.40 \\
\hline 3448.32 & 5572.48 & 5696.15 & 3304.17 & 125.22 \\
\hline 3449.99 & 5469.02 & 5359.96 & 3229.86 & 123.95 \\
\hline 3451.65 & 5408.80 & 5207.03 & 3175.12 & 109.67 \\
\hline 3453.32 & 5256.49 & 4927.15 & 3107.91 & 123.29 \\
\hline 3454.98 & 5242.17 & 4795.26 & 3057.23 & 141.09 \\
\hline 3456.65 & 5102.66 & 4428.41 & 2961.25 & 148.81 \\
\hline 3458.31 & 5008.00 & 4371.34 & 2923.37 & 135.65 \\
\hline 3459.97 & 4973.88 & 4214.61 & 2883.59 & 126.86 \\
\hline 3461.64 & 4837.92 & 4003.06 & 2812.35 & 171.29 \\
\hline
\end{tabular}




\section{Supplementary Information and Figures}

\begin{tabular}{|c|c|c|c|c|}
\hline 3463.30 & 4746.61 & 4012.52 & 2768.54 & 151.78 \\
\hline 3464.96 & 4675.30 & 3807.68 & 2704.26 & 139.15 \\
\hline 3466.62 & 4539.24 & 3700.09 & 2652.95 & 148.29 \\
\hline 3468.28 & 4453.48 & 3625.00 & 2593.43 & 154.89 \\
\hline 3469.94 & 4339.07 & 3461.89 & 2533.09 & 154.91 \\
\hline 3471.60 & 4282.71 & 3443.51 & 2515.21 & 111.30 \\
\hline 3473.26 & 4208.76 & 3288.03 & 2448.40 & 147.85 \\
\hline 3474.91 & 4124.95 & 3209.61 & 2411.18 & 135.83 \\
\hline 3476.57 & 4061.79 & 3200.28 & 2393.47 & 133.42 \\
\hline 3478.23 & 3955.89 & 3044.80 & 2342.69 & 134.07 \\
\hline 3479.88 & 3908.93 & 2931.71 & 2300.18 & 152.55 \\
\hline 3481.54 & 3764.88 & 2679.71 & 2243.00 & 129.29 \\
\hline 3483.19 & 3719.83 & 2617.29 & 2216.45 & 122.89 \\
\hline 3484.85 & 3644.82 & 2568.40 & 2192.20 & 159.76 \\
\hline 3486.50 & 3547.27 & 2488.37 & 2156.86 & 119.85 \\
\hline 3488.15 & 3479.82 & 2277.23 & 2108.94 & 156.90 \\
\hline 3489.80 & 3388.02 & 2163.52 & 2086.06 & 120.97 \\
\hline 3491.45 & 3377.02 & 2129.49 & 2056.48 & 122.25 \\
\hline 3493.10 & 3292.22 & 2082.61 & 2040.20 & 152.19 \\
\hline 3494.75 & 3159.07 & 2049.62 & 2022.22 & 136.80 \\
\hline 3496.40 & 3123.88 & 1938.64 & 1984.84 & 120.11 \\
\hline 3498.05 & 3038.58 & 1734.29 & 1945.39 & 88.89 \\
\hline 3499.70 & 2940.44 & 1763.75 & 1935.97 & 112.79 \\
\hline 3501.35 & 2875.44 & 1681.14 & 1910.93 & 111.39 \\
\hline 3502.99 & 2793.80 & 1575.95 & 1879.81 & 112.46 \\
\hline 3504.64 & 2677.60 & 1625.10 & 1898.06 & 88.69 \\
\hline 3506.28 & 2673.61 & 1545.27 & 1857.15 & 125.23 \\
\hline 3507.93 & 2580.37 & 1502.29 & 1851.17 & 114.72 \\
\hline 3509.57 & 2548.88 & 1562.08 & 1845.58 & 120.48 \\
\hline 3511.22 & 2463.94 & 1314.06 & 1804.17 & 99.64 \\
\hline 3512.86 & 2380.95 & 1471.13 & 1835.02 & 105.36 \\
\hline 3514.50 & 2348.51 & 1373.21 & 1803.37 & 105.04 \\
\hline 3516.14 & 2285.82 & 1393.55 & 1807.72 & 93.98 \\
\hline 3517.78 & 2189.58 & 1162.15 & 1766.24 & 107.92 \\
\hline 3519.42 & 2208.60 & 1343.49 & 1794.59 & 128.12 \\
\hline 3521.06 & 2108.76 & 1162.56 & 1774.24 & 117.73 \\
\hline 3522.70 & 2091.98 & 1178.20 & 1786.19 & 163.54 \\
\hline 3524.34 & 2004.84 & 1150.23 & 1768.87 & 124.85 \\
\hline 3525.98 & 1996.06 & 974.03 & 1766.59 & 144.37 \\
\hline 3527.62 & 1933.23 & 1028.48 & 1758.11 & 141.79 \\
\hline 3529.25 & 1866.45 & 999.99 & 1752.79 & 135.38 \\
\hline 3530.89 & 1804.42 & 1065.36 & 1769.91 & 144.20 \\
\hline 3532.52 & 1782.94 & 1010.07 & 1753.42 & 162.14 \\
\hline 3534.16 & 1688.76 & 1047.23 & 1736.67 & 157.07 \\
\hline
\end{tabular}




\section{Supplementary Information and Figures}

\begin{tabular}{|c|c|c|c|c|}
\hline 3535.79 & 1687.53 & 911.32 & 1715.26 & 144.96 \\
\hline 3537.43 & 1609.66 & 916.08 & 1684.30 & 159.45 \\
\hline 3539.06 & 1578.63 & 937.81 & 1678.49 & 161.49 \\
\hline 3540.69 & 1529.81 & 910.07 & 1656.14 & 146.93 \\
\hline 3542.32 & 1479.08 & 763.31 & 1588.65 & 163.57 \\
\hline 3543.95 & 1466.41 & 714.57 & 1566.70 & 151.76 \\
\hline 3545.58 & 1387.39 & 714.65 & 1540.38 & 137.60 \\
\hline 3547.21 & 1377.82 & 743.02 & 1516.36 & 107.18 \\
\hline 3548.84 & 1356.35 & 613.20 & 1468.65 & 122.51 \\
\hline 3550.47 & 1291.73 & 604.49 & 1434.06 & 113.24 \\
\hline 3552.10 & 1288.11 & 559.59 & 1412.18 & 130.72 \\
\hline 3553.72 & 1251.39 & 636.86 & 1388.66 & 55.30 \\
\hline 3555.35 & 1193.42 & 632.43 & 1356.77 & 65.82 \\
\hline 3556.98 & 1166.16 & 502.06 & 1320.19 & 93.14 \\
\hline 3558.60 & 1145.34 & 529.83 & 1295.00 & 64.45 \\
\hline 3560.22 & 1109.03 & 433.37 & 1250.35 & 74.71 \\
\hline 3561.85 & 1087.42 & 344.28 & 1224.30 & 69.01 \\
\hline 3563.47 & 1057.91 & 514.74 & 1249.38 & 94.41 \\
\hline 3565.09 & 1031.54 & 477.40 & 1201.29 & 137.00 \\
\hline 3566.72 & 992.84 & 486.57 & 1187.81 & 129.43 \\
\hline 3568.34 & 985.18 & 399.79 & 1127.46 & 112.31 \\
\hline 3569.96 & 968.77 & 359.11 & 1108.54 & 101.93 \\
\hline 3571.58 & 962.66 & 497.61 & 1114.68 & 86.99 \\
\hline 3573.20 & 922.06 & 437.04 & 1066.56 & 105.34 \\
\hline 3574.82 & 860.30 & 494.44 & 1055.18 & 134.28 \\
\hline 3576.43 & 871.50 & 499.54 & 1029.99 & 137.32 \\
\hline 3578.05 & 857.14 & 441.06 & 1001.20 & 172.09 \\
\hline 3579.67 & 846.54 & 308.60 & 969.98 & 158.10 \\
\hline 3581.28 & 805.14 & 318.51 & 934.93 & 199.51 \\
\hline 3582.90 & 830.99 & 382.60 & 935.28 & 169.25 \\
\hline 3584.52 & 785.64 & 328.34 & 901.92 & 149.82 \\
\hline 3586.13 & 774.35 & 506.14 & 905.47 & 155.00 \\
\hline 3587.74 & 743.25 & 452.60 & 876.91 & 201.61 \\
\hline 3589.36 & 746.76 & 441.91 & 860.06 & 148.39 \\
\hline 3590.97 & 722.21 & 490.48 & 846.07 & 168.27 \\
\hline 3592.58 & 671.52 & 373.06 & 810.62 & 162.19 \\
\hline 3594.19 & 705.12 & 472.90 & 787.96 & 153.65 \\
\hline 3595.80 & 681.78 & 381.48 & 757.68 & 152.58 \\
\hline 3597.41 & 639.39 & 421.75 & 750.75 & 157.31 \\
\hline 3599.02 & 646.45 & 486.52 & 756.73 & 143.56 \\
\hline 3600.63 & 594.77 & 455.19 & 712.78 & 124.45 \\
\hline 3602.24 & 599.73 & 393.71 & 689.99 & 134.03 \\
\hline 3603.85 & 571.54 & 325.45 & 661.20 & 173.49 \\
\hline 3605.45 & 541.11 & 203.92 & 621.68 & 156.58 \\
\hline
\end{tabular}




\section{Supplementary Information and Figures}

\begin{tabular}{|c|c|c|c|c|}
\hline 3607.06 & 535.73 & 294.62 & 619.69 & 150.56 \\
\hline 3608.66 & 488.19 & 377.88 & 625.24 & 181.92 \\
\hline 3610.27 & 507.61 & 331.20 & 593.85 & 158.40 \\
\hline 3611.87 & 517.78 & 313.30 & 574.33 & 137.28 \\
\hline 3613.48 & 478.20 & 376.86 & 581.97 & 127.67 \\
\hline 3615.08 & 501.57 & 178.83 & 527.21 & 118.71 \\
\hline 3616.68 & 466.10 & 370.81 & 530.86 & 164.82 \\
\hline 3618.29 & 444.37 & 334.06 & 514.97 & 171.26 \\
\hline 3619.89 & 416.30 & 231.32 & 482.95 & 169.93 \\
\hline 3621.49 & 440.42 & 183.40 & 455.12 & 131.73 \\
\hline 3623.09 & 419.35 & 265.05 & 459.94 & 182.11 \\
\hline 3624.69 & 411.03 & 253.52 & 441.78 & 185.46 \\
\hline 3626.28 & 395.11 & 366.32 & 450.59 & 182.06 \\
\hline 3627.88 & 397.09 & 387.94 & 430.17 & 172.37 \\
\hline 3629.48 & 388.97 & 392.98 & 426.08 & 179.56 \\
\hline 3631.08 & 385.31 & 412.03 & 413.75 & 173.42 \\
\hline 3632.67 & 384.39 & 339.30 & 391.10 & 184.22 \\
\hline 3634.27 & 339.28 & 226.59 & 356.57 & 172.03 \\
\hline 3635.86 & 328.76 & 365.00 & 363.25 & 154.68 \\
\hline 3637.46 & 344.55 & 294.52 & 347.13 & 166.69 \\
\hline 3639.05 & 349.49 & 203.90 & 321.40 & 160.79 \\
\hline 3640.64 & 321.23 & 303.76 & 319.11 & 150.91 \\
\hline 3642.24 & 310.23 & 391.25 & 326.42 & 146.89 \\
\hline 3643.83 & 304.87 & 344.39 & 311.30 & 147.66 \\
\hline 3645.42 & 311.56 & 399.09 & 312.24 & 150.55 \\
\hline 3647.01 & 264.16 & 183.00 & 274.35 & 111.91 \\
\hline 3648.60 & 301.91 & 211.06 & 265.52 & 122.84 \\
\hline 3650.19 & 269.70 & 354.68 & 280.37 & 148.30 \\
\hline 3651.78 & 219.25 & 373.16 & 276.67 & 124.32 \\
\hline 3653.37 & 256.30 & 370.79 & 263.38 & 149.37 \\
\hline 3654.95 & 219.85 & 348.19 & 256.16 & 136.70 \\
\hline 3656.54 & 241.11 & 199.53 & 223.27 & 144.53 \\
\hline 3658.13 & 217.86 & 196.24 & 225.21 & 150.23 \\
\hline 3659.71 & 240.27 & 305.39 & 233.08 & 139.07 \\
\hline 3661.30 & 210.17 & 378.59 & 227.49 & 165.01 \\
\hline 3662.88 & 190.83 & 370.78 & 237.63 & 141.23 \\
\hline 3664.47 & 159.94 & 311.76 & 209.44 & 118.41 \\
\hline 3666.05 & 123.00 & 321.08 & 203.88 & 102.82 \\
\hline 3667.63 & 208.16 & 226.11 & 197.56 & 119.64 \\
\hline 3669.21 & 165.78 & 389.91 & 211.70 & 153.19 \\
\hline 3670.80 & 182.24 & 183.86 & 173.47 & 116.79 \\
\hline 3672.38 & 164.16 & 149.88 & 166.08 & 119.92 \\
\hline 3673.96 & 154.32 & 232.19 & 164.99 & 106.22 \\
\hline 3675.54 & 181.24 & 323.54 & 172.83 & 109.69 \\
\hline
\end{tabular}




\section{Supplementary Information and Figures}

\begin{tabular}{|c|c|c|c|c|}
\hline 3677.11 & 141.36 & 355.51 & 181.87 & 98.01 \\
\hline 3678.69 & 148.98 & 355.13 & 169.68 & 146.42 \\
\hline 3680.27 & 148.15 & 213.80 & 150.22 & 125.06 \\
\hline 3681.85 & 107.08 & 369.18 & 175.06 & 110.62 \\
\hline 3683.42 & 146.10 & 355.05 & 167.53 & 123.67 \\
\hline 3685.00 & 134.78 & 401.70 & 163.54 & 151.33 \\
\hline 3686.57 & 130.05 & 260.71 & 142.21 & 135.63 \\
\hline 3688.15 & 148.28 & 327.81 & 149.35 & 132.88 \\
\hline 3689.72 & 130.56 & 389.50 & 157.02 & 116.53 \\
\hline 3691.30 & 177.05 & 289.67 & 132.43 & 136.24 \\
\hline 3692.87 & 103.18 & 285.95 & 133.57 & 134.44 \\
\hline 3694.44 & 122.76 & 396.65 & 147.64 & 171.42 \\
\hline 3696.01 & 123.50 & 227.73 & 112.42 & 168.18 \\
\hline 3697.58 & 130.23 & 266.40 & 114.19 & 163.01 \\
\hline 3699.15 & 110.52 & 324.43 & 112.66 & 141.32 \\
\hline 3700.72 & 110.21 & 320.79 & 113.50 & 156.01 \\
\hline 3702.29 & 145.30 & 293.55 & 109.97 & 157.78 \\
\hline 3703.86 & 81.04 & 318.79 & 106.78 & 172.38 \\
\hline 3705.43 & 118.14 & 369.38 & 116.65 & 134.21 \\
\hline 3706.99 & 105.23 & 377.14 & 112.69 & 134.64 \\
\hline 3708.56 & 106.23 & 399.83 & 122.50 & 147.43 \\
\hline 3710.13 & 88.63 & 363.58 & 110.00 & 147.26 \\
\hline 3711.69 & 84.17 & 364.92 & 109.48 & 126.68 \\
\hline 3713.26 & 98.38 & 350.49 & 100.75 & 100.77 \\
\hline 3714.82 & 109.33 & 313.42 & 97.42 & 141.87 \\
\hline 3716.38 & 102.53 & 363.38 & 105.09 & 117.34 \\
\hline 3717.95 & 114.49 & 376.29 & 102.00 & 106.33 \\
\hline 3719.51 & 78.64 & 418.21 & 138.40 & 103.53 \\
\hline 3721.07 & 80.50 & 213.64 & 81.87 & 119.81 \\
\hline 3722.63 & 99.36 & 356.33 & 100.51 & 108.93 \\
\hline 3724.19 & 78.67 & 302.05 & 89.78 & 157.59 \\
\hline 3725.75 & 81.98 & 425.95 & 99.65 & 125.53 \\
\hline 3727.31 & 75.44 & 349.26 & 94.89 & 137.91 \\
\hline 3728.87 & 80.31 & 206.30 & 66.80 & 157.72 \\
\hline 3730.43 & 64.32 & 330.10 & 87.50 & 136.93 \\
\hline 3731.98 & 103.14 & 257.46 & 81.24 & 117.64 \\
\hline 3733.54 & 68.01 & 196.42 & 68.31 & 107.18 \\
\hline 3735.10 & 69.03 & 182.89 & 61.28 & 80.90 \\
\hline 3736.65 & 89.30 & 391.59 & 118.62 & 86.93 \\
\hline 3738.21 & 49.03 & 319.62 & 87.12 & 81.24 \\
\hline 3739.76 & 62.50 & 313.86 & 86.59 & 75.06 \\
\hline 3741.31 & 53.77 & 193.17 & 62.00 & 93.12 \\
\hline 3742.87 & 75.40 & 294.63 & 80.57 & 90.55 \\
\hline 3744.42 & 66.23 & 307.34 & 76.50 & 109.74 \\
\hline
\end{tabular}


Supplementary Information and Figures

\begin{tabular}{|c|c|c|c|c|}
\hline 3745.97 & 94.71 & 244.24 & 70.61 & 100.83 \\
\hline 3747.52 & 87.74 & 329.73 & 80.41 & 119.71 \\
\hline 3749.07 & 55.18 & 359.30 & 91.35 & 121.19 \\
\hline 3750.62 & 83.51 & 229.21 & 63.35 & 92.41 \\
\hline 3752.17 & 85.95 & 316.95 & 77.12 & 114.13 \\
\hline 3753.72 & 62.44 & 252.00 & 65.69 & 127.69 \\
\hline 3755.27 & 55.43 & 330.66 & 72.03 & 124.50 \\
\hline 3756.82 & 52.32 & 264.06 & 69.46 & 105.55 \\
\hline 3758.36 & 73.11 & 273.79 & 61.87 & 169.98 \\
\hline 3759.91 & 66.40 & 399.78 & 88.64 & 96.93 \\
\hline 3761.45 & 54.45 & 298.12 & 66.31 & 143.30 \\
\hline 3763.00 & 86.49 & 206.84 & 53.11 & 131.09 \\
\hline 3764.54 & 65.34 & 377.84 & 70.48 & 137.91 \\
\hline 3766.09 & 65.49 & 279.48 & 63.28 & 153.16 \\
\hline 3767.63 & 73.84 & 345.19 & 67.02 & 144.62 \\
\hline 3769.17 & 47.45 & 294.05 & 59.48 & 139.54 \\
\hline 3770.72 & 71.25 & 278.17 & 61.59 & 135.14 \\
\hline 3772.26 & 84.71 & 238.09 & 48.66 & 136.46 \\
\hline 3773.80 & 119.92 & 450.66 & 78.86 & 155.38 \\
\hline 3775.34 & 64.12 & 368.03 & 61.33 & 189.01 \\
\hline 3776.88 & 92.98 & 315.97 & 65.10 & 148.73 \\
\hline 3778.42 & 83.20 & 363.76 & 65.53 & 141.67 \\
\hline 3779.95 & 91.31 & 415.11 & 74.93 & 120.52 \\
\hline 3781.49 & 72.87 & 278.36 & 48.70 & 146.56 \\
\hline 3783.03 & 55.29 & 314.18 & 52.70 & 172.61 \\
\hline 3784.57 & 100.71 & 213.19 & 44.57 & 133.51 \\
\hline 3786.10 & 74.08 & 324.15 & 60.27 & 118.53 \\
\hline 3787.64 & 72.30 & 266.15 & 54.68 & 159.99 \\
\hline 3789.17 & 63.12 & 331.45 & 61.11 & 171.83 \\
\hline 3790.70 & 63.39 & 247.85 & 49.94 & 144.08 \\
\hline 3792.24 & 67.62 & 371.67 & 73.45 & 151.91 \\
\hline 3793.77 & 32.99 & 230.31 & 51.25 & 144.82 \\
\hline 3795.30 & 37.77 & 290.68 & 55.28 & 131.69 \\
\hline 3796.84 & 114.10 & 223.08 & 40.28 & 126.10 \\
\hline 3798.37 & 27.63 & 289.07 & 58.65 & 158.94 \\
\hline 3799.90 & 46.46 & 152.47 & 46.98 & 138.34 \\
\hline 3801.43 & 45.89 & 204.29 & 47.55 & 151.50 \\
\hline 3802.96 & 81.27 & 198.58 & 47.89 & 113.02 \\
\hline 3804.48 & 77.01 & 121.19 & 32.45 & 114.27 \\
\hline 3806.01 & 40.44 & 343.88 & 64.25 & 121.06 \\
\hline 3807.54 & 93.88 & 257.47 & 57.72 & 118.42 \\
\hline 3809.07 & 61.32 & 278.46 & 58.46 & 116.38 \\
\hline 3810.59 & 66.11 & 196.11 & 49.59 & 96.88 \\
\hline 3812.12 & 12.65 & 267.28 & 56.22 & 152.22 \\
\hline
\end{tabular}


Supplementary Information and Figures

\begin{tabular}{|c|c|c|c|c|}
\hline 3813.64 & 77.79 & 120.37 & 33.86 & 113.45 \\
\hline 3815.17 & 81.63 & 210.25 & 49.52 & 111.81 \\
\hline 3816.69 & 64.42 & 194.79 & 40.59 & 123.26 \\
\hline 3818.21 & 83.27 & 199.72 & 45.69 & 143.02 \\
\hline 3819.74 & 45.67 & 263.49 & 45.86 & 144.37 \\
\hline 3821.26 & 52.56 & 260.63 & 47.72 & 161.86 \\
\hline 3822.78 & 53.86 & 306.29 & 70.89 & 144.65 \\
\hline 3824.30 & 41.11 & 303.91 & 60.99 & 146.86 \\
\hline 3825.82 & 51.56 & 208.72 & 34.19 & 147.38 \\
\hline 3827.34 & 101.76 & 205.92 & 44.16 & 123.81 \\
\hline 3828.86 & 65.96 & 298.65 & 55.29 & 145.55 \\
\hline 3830.38 & 55.61 & 349.27 & 72.52 & 143.25 \\
\hline 3831.89 & 80.66 & 347.51 & 70.82 & 172.48 \\
\hline 3833.41 & 46.27 & 375.16 & 60.89 & 162.60 \\
\hline 3834.93 & 55.08 & 235.32 & 49.19 & 142.18 \\
\hline 3836.44 & 93.58 & 157.94 & 41.78 & 145.05 \\
\hline 3837.96 & 51.84 & 359.41 & 83.02 & 142.30 \\
\hline 3839.47 & 99.60 & 190.44 & 35.68 & 144.09 \\
\hline 3840.99 & 76.81 & 303.98 & 53.85 & 152.41 \\
\hline 3842.50 & 41.57 & 381.91 & 63.21 & 165.47 \\
\hline 3844.01 & 82.73 & 370.10 & 63.65 & 154.48 \\
\hline 3845.53 & 64.14 & 242.20 & 46.91 & 144.49 \\
\hline 3847.04 & 90.40 & 242.64 & 49.64 & 166.97 \\
\hline 3848.55 & 86.72 & 333.43 & 58.88 & 156.52 \\
\hline 3850.06 & 57.88 & 362.29 & 59.54 & 128.37 \\
\hline 3851.57 & 60.14 & 265.88 & 46.24 & 143.43 \\
\hline 3853.08 & 67.46 & 280.60 & 41.67 & 160.41 \\
\hline 3854.59 & 119.88 & 250.77 & 39.60 & 139.84 \\
\hline 3856.10 & 69.79 & 276.89 & 47.70 & 137.63 \\
\hline 3857.60 & 34.41 & 257.68 & 55.70 & 139.20 \\
\hline 3859.11 & 66.58 & 391.45 & 72.60 & 144.71 \\
\hline 3860.62 & 91.30 & 401.47 & 73.16 & 122.73 \\
\hline 3862.12 & 97.87 & 351.14 & 68.66 & 118.37 \\
\hline 3863.63 & 52.29 & 407.87 & 65.46 & 85.71 \\
\hline 3865.13 & 53.71 & 351.53 & 57.79 & 98.57 \\
\hline 3866.64 & 98.63 & 324.99 & 49.69 & 107.98 \\
\hline 3868.14 & 56.45 & 368.44 & 67.09 & 108.31 \\
\hline 3869.64 & 79.67 & 331.13 & 50.42 & 141.70 \\
\hline 3871.14 & 70.60 & 223.83 & 30.35 & 140.19 \\
\hline 3872.65 & 69.42 & 384.94 & 86.25 & 137.18 \\
\hline 3874.15 & 49.85 & 362.16 & 68.28 & 142.39 \\
\hline 3875.65 & 61.17 & 368.60 & 58.91 & 132.52 \\
\hline 3877.15 & 79.50 & 284.21 & 48.60 & 131.84 \\
\hline 3878.65 & 39.27 & 347.86 & 90.74 & 126.33 \\
\hline
\end{tabular}


Supplementary Information and Figures

\begin{tabular}{|c|c|c|c|c|}
\hline 3880.14 & 89.35 & 375.55 & 58.97 & 136.59 \\
\hline 3881.64 & 55.82 & 302.42 & 54.66 & 97.14 \\
\hline 3883.14 & 68.10 & 233.07 & 32.89 & 131.50 \\
\hline 3884.64 & 33.38 & 307.20 & 52.62 & 114.77 \\
\hline 3886.13 & 51.61 & 174.62 & 37.05 & 121.96 \\
\hline 3887.63 & 59.09 & 176.20 & 25.75 & 95.19 \\
\hline 3889.12 & 63.87 & 160.50 & 30.58 & 92.50 \\
\hline 3890.62 & 92.45 & 330.28 & 59.61 & 116.24 \\
\hline 3892.11 & 76.13 & 217.62 & 42.91 & 102.11 \\
\hline 3893.60 & 63.21 & 152.56 & 23.47 & 127.54 \\
\hline 3895.10 & 70.44 & 133.90 & 31.87 & 102.30 \\
\hline 3896.59 & 47.92 & 273.70 & 49.70 & 130.24 \\
\hline 3898.08 & 34.40 & 294.65 & 44.46 & 103.34 \\
\hline 3899.57 & 49.73 & 276.43 & 49.16 & 129.04 \\
\hline 3901.06 & 83.61 & 213.37 & 46.12 & 152.25 \\
\hline 3902.55 & 53.90 & 290.75 & 59.82 & 141.66 \\
\hline 3904.04 & 28.53 & 303.53 & 51.48 & 118.93 \\
\hline 3905.53 & 78.31 & 160.23 & 28.38 & 138.41 \\
\hline 3907.01 & 44.24 & 341.65 & 72.74 & 161.81 \\
\hline 3908.50 & 43.73 & 303.40 & 49.17 & 104.55 \\
\hline 3909.99 & 57.76 & 268.88 & 49.27 & 152.04 \\
\hline 3911.47 & 56.75 & 278.97 & 45.26 & 136.86 \\
\hline 3912.96 & 66.03 & 242.31 & 52.06 & 150.21 \\
\hline 3914.44 & 41.81 & 295.98 & 51.99 & 166.24 \\
\hline 3915.93 & 62.80 & 177.51 & 35.22 & 162.01 \\
\hline 3917.41 & 62.13 & 230.69 & 46.81 & 127.55 \\
\hline 3918.89 & 83.32 & 256.85 & 44.97 & 172.69 \\
\hline 3920.38 & 37.71 & 317.44 & 54.17 & 116.62 \\
\hline 3921.86 & 54.39 & 335.00 & 62.90 & 129.77 \\
\hline 3923.34 & 96.48 & 371.55 & 74.63 & 110.38 \\
\hline 3924.82 & 76.61 & 277.61 & 55.59 & 112.69 \\
\hline 3926.30 & 45.75 & 327.85 & 76.88 & 121.56 \\
\hline 3927.78 & 68.88 & 267.77 & 52.75 & 99.88 \\
\hline 3929.26 & 58.07 & 342.60 & 85.48 & 138.31 \\
\hline 3930.74 & 56.81 & 232.74 & 55.37 & 111.84 \\
\hline 3932.21 & 76.69 & 200.04 & 40.73 & 101.59 \\
\hline 3933.69 & 49.43 & 251.05 & 76.23 & 99.08 \\
\hline 3935.17 & 55.01 & 148.98 & 43.29 & 91.27 \\
\hline 3936.64 & 35.40 & 150.16 & 43.75 & 124.24 \\
\hline 3938.12 & 39.58 & 168.85 & 54.51 & 107.04 \\
\hline 3939.59 & 35.62 & 190.20 & 52.34 & 90.71 \\
\hline 3941.07 & 49.51 & 140.14 & 49.37 & 93.72 \\
\hline 3942.54 & 26.49 & 30.74 & 35.80 & 122.80 \\
\hline 3944.01 & 42.58 & 72.15 & 33.26 & 126.38 \\
\hline
\end{tabular}


Supplementary Information and Figures

\begin{tabular}{|c|c|c|c|c|}
\hline 3945.49 & 74.16 & 107.21 & 48.39 & 115.05 \\
\hline 3946.96 & 67.60 & 210.24 & 70.28 & 95.99 \\
\hline 3948.43 & 43.53 & 41.53 & 28.41 & 121.78 \\
\hline 3949.90 & 65.77 & 11.36 & 34.77 & 129.03 \\
\hline 3951.37 & 61.86 & 168.77 & 77.23 & 126.82 \\
\hline 3952.84 & 27.94 & 9.44 & 37.19 & 102.78 \\
\hline 3954.31 & 54.18 & 207.40 & 69.89 & 115.43 \\
\hline 3955.78 & 52.71 & 139.04 & 50.88 & 104.09 \\
\hline 3957.24 & 48.85 & 250.43 & 101.24 & 89.51 \\
\hline 3958.71 & 91.18 & 111.43 & 44.23 & 86.73 \\
\hline 3960.18 & 80.32 & 182.55 & 54.96 & 149.74 \\
\hline 3961.64 & 120.15 & 134.71 & 50.09 & 101.38 \\
\hline 3963.11 & 72.38 & 178.64 & 55.45 & 132.10 \\
\hline 3964.57 & 30.72 & 184.71 & 59.48 & 131.34 \\
\hline 3966.04 & 68.50 & 119.88 & 53.51 & 133.46 \\
\hline 3967.50 & 55.79 & 196.34 & 66.10 & 135.15 \\
\hline 3968.96 & 39.77 & 169.56 & 68.06 & 167.80 \\
\hline 3970.42 & 75.30 & 186.62 & 62.52 & 162.14 \\
\hline 3971.89 & 73.19 & 178.55 & 61.71 & 112.23 \\
\hline 3973.35 & 83.77 & 220.78 & 73.14 & 135.33 \\
\hline 3974.81 & 63.95 & 122.10 & 50.57 & 150.38 \\
\hline 3976.27 & 103.68 & 191.50 & 54.39 & 153.98 \\
\hline 3977.73 & 52.06 & 123.67 & 56.95 & 163.64 \\
\hline 3979.19 & 48.35 & 261.90 & 76.41 & 178.45 \\
\hline 3980.64 & 100.17 & 208.31 & 65.24 & 163.82 \\
\hline 3982.10 & 63.96 & 261.17 & 75.73 & 156.18 \\
\hline 3983.56 & 80.19 & 272.96 & 95.26 & 143.99 \\
\hline 3985.01 & 82.16 & 298.10 & 77.39 & 177.22 \\
\hline 3986.47 & 82.59 & 99.53 & 51.98 & 181.03 \\
\hline 3987.93 & 88.33 & 98.38 & 48.87 & 131.99 \\
\hline 3989.38 & 101.65 & 177.75 & 56.57 & 150.77 \\
\hline 3990.83 & 85.43 & 264.95 & 68.76 & 156.95 \\
\hline 3992.29 & 91.16 & 335.71 & 90.55 & 133.87 \\
\hline 3993.74 & 88.69 & 175.48 & 59.11 & 125.65 \\
\hline 3995.19 & 91.21 & 248.73 & 60.60 & 196.53 \\
\hline 3996.64 & 54.29 & 393.16 & 85.33 & 153.12 \\
\hline 3998.10 & 69.47 & 319.53 & 64.86 & 154.08 \\
\hline 3999.55 & 58.59 & 212.35 & 55.12 & 131.12 \\
\hline 4001.00 & 111.67 & 392.69 & 85.71 & 140.60 \\
\hline 4002.44 & 111.64 & 240.00 & 50.00 & 120.00 \\
\hline
\end{tabular}

End of Supplementary Information 